\title{
Essai d'amélioration des rendements du Pin Sylvestre et du Pin de Corse sur sols sableux (Podzol)
}

\author{
PAR \\ J. - C. BERBEN \\ Ingénieur des Eaux et Forêts U.C.L. \\ Centre de Recherches en Biologie Forestière, Bokrijk-Genk* \\ et
}

\section{A. LENGER}

Ingénieur des Eaux et Foréts A.I.Gx.

Bureau de Biométrie, Bruxelles* 



\section{1 - Introduction.}

Depuis le milieu du siècle dernier, la politique forestière en Campine est orientée vers la production de bois de mine par une exploitation extensive des sols. Les peuplements sont en majeure partie des monocultures de pins sylvestres ou de pins de Corse: comme le montrent les statistiques de $1959\left({ }^{*}\right)$ reproduites ci-après, ces deux essences occupent à elles seules dans les provinces d'Anvers et du Limbourg, respectivement 62 et $79 \%$ de la superficie boisée; cependant, le pin de Corse, d'introduction plus récente, est moins largement représenté que le pin sylvestre.

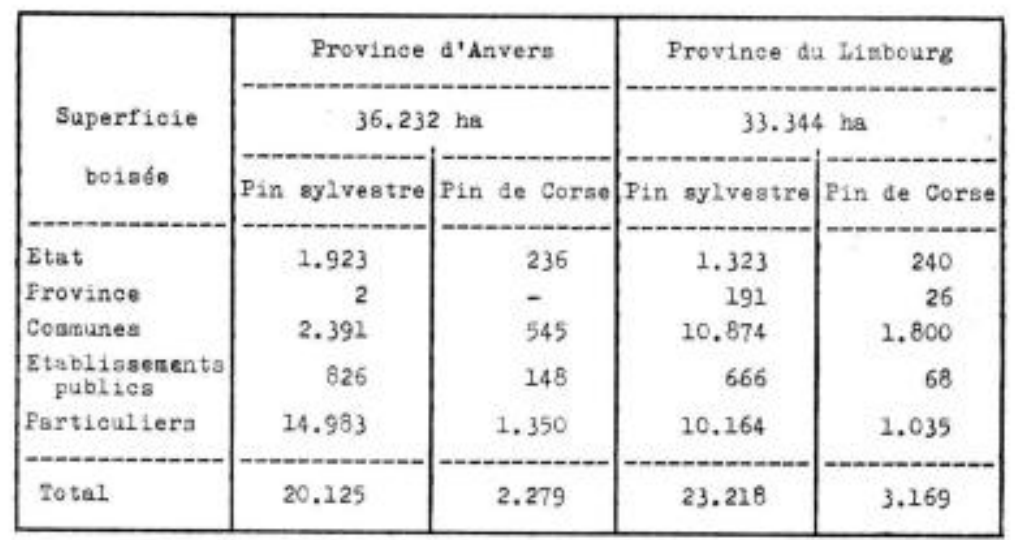

Les deux impératifs suivants ont dicté, en ordre principal, cette politique forestière devenue traditionnelle:

1) la pauvreté et la faible capacité de rétention en cau des sols sablonneux de Campine qui limitent le choix des essences,

2) le développement des charbonnages de la région entre les deux guerres mondiales, off rant sur place un débouché important aux produits de la sylviculture.

* Subsidiés par l'Institut pour l'Encouragement de la Recherche Scientifique dans l'Industric et l'Agriculture (I.R.S.I.A.),

** Algemene landbouw- en Bostelling van 1959, Ministerie van Ekonomische Zaken en Energie, Boekdeel V, Brussel 1962. 
Malheureusement, ces peuplements de pins sylvestres et de pins de Corse ont des rendement en général médiocres. Les inventaires successifs effectués par la Station de Recherches de Groenendael dans un réseau étendu de parcelles de production, font apparaitre pour un terme de 40 à 45 ans, un accroissement annuel moyen par ha de 3 à $5 \mathrm{~m}^{3}$ pour le pin sylvestre en Hate Campine, de 4 à $5,5 \mathrm{~m}^{3}$ pour cette mème essence en Basse Campine, et de 8 à $9 \mathrm{~m}^{3}$ pour le pin de Corse. Ces valeurs sont supérieures aux rendements moyens réels: il semble en effet que la forêt campinoise compte dans son ensemble, proportionnellement plus de parcelles à faible production que le réseau expérimental (GALoux, 1952).

D'autre part, ces types de peuplements ne répondent plus parfaitement aux exigences économiques actuelles. La valeur des fonds et les frais de production et d'exploitation ne cessent d'augmenter dans des proportions telles qu'une exploitation extensive de faible production risque de ne plus être rentable dans un proche avenir. Il faut de plus trouver d'autres débouchés que ceux offerts par les charbonnages et par conséquent, diversifier davantage les produits et en améliorer les qualités technologiques.

Enfin, il faut pallier les inconvénients des monocultures (épuisement du sol, conditions phytosanitaires défavorables, incendies...) susceptibles de réduire ou de déprécier une production déjà faible en volume.

Compte tenu de ces éléments, la Section de sylviculture du Centre de Recherches en Biologie Forestière de Bokrijk poursuit actuellement deux buts:

1) Mise at point de méthodes d'exploitation intensive par l'utilisation rationnelle des sols: travail du sol, amendements, choix des essences.

2) Mise au point de techniques culturales: modes de plantation, ćcartements, mélanges d'espèces, rythme et intensité des éclaircies.

Il est normal que la première tâche de la Section consiste à améliorer la rentabilité des deux essences traditionnelles de Campine, le pin sylvestre et le pin de Corse, qui sont bien adaptées aux substrats très secs généralement rencontrés dans cette région.

Ceci n'exclut pas les essais d'introduction d'espèces résineuses nouvelles, intéressantes pour la qualité de leur bois et la vigueur de leur croissance, et d'essences feuillues à retenir pour des raisons culturales ou phytosanitaires. L'orientation des essais entrepris à cet égard par la Section, est essentiellement dictée par les données concrètes de la littérature étrangère (Duchaufour, 1958; LeIBundgut et Kreutzer, 1958; Schelling, 1960; Schwerdtfeger, 1957). 
Nous présentons ici les premiers résultats de deux expériences réalisées sur pin sylvestre et pin de Corse à Mechelen-aan-de-Maas relevant de la première tâche que s'est assignée le Centre de Bokrijk.

Cette recherche a été entreprise en collaboration par le Centre de Bokrijk et le Bureau de Biométrie, subsidiés l'un et l'autre par I'I.R.S.I.A. : les protocoles expérimentaux, l'exécution des essais et l'interprétation biologique des résultats sont le fait du Centre de Bokrijk; la planification des expériences, l'analyse et l'interprétation statistiques sont réalisées par le Bureau de Biométrie.

F. Dufrane, titulaire de la Section à l'origine, est le promoteur de cette recherche; les auteurs sont responsables, l'un de la direction des travaux et de la récolte des données (J. B.), l'autre de l'intervention statistique (A. L.); les données pédologiques ont été fournies par D. LAMBERTs (LAмвERTs, 1958), chef de travaux de la Section de Pédologie du Centre Forestier de Bokrijk.

\section{2 - Buts des expériences.}

\section{1 - Expérience sur pin sylvestre.}

Cette expérience a pour but de contrôler l'effet d'une fumure (2 tonnes de marne +1 tonne de scories de déphosphoration par ha, épandues en plein avant le travail du sol, et $200 \mathrm{~kg}$ de chlorure de potasse $20 \%+100 \mathrm{~kg}$ de nitrate d'ammoniaque $17 \%$ par ha, épandus en plein un an après la plantation) et de trois écartements de plantation $(1 \mathrm{~m} \times 1 \mathrm{~m}, 1 \mathrm{~m} \times 2 \mathrm{~m}, 2 \mathrm{~m} \times 2 \mathrm{~m})$ sur la croissance du pin sylvestre.

Dans cette expérience, le champ d'essai a subi un travail du sol uniforme: fraisage par bandes de $50 \mathrm{~cm}$ de largeur après soussolage à $60 \mathrm{~cm}$ de profondeur, suivant l'axe des bandes.

\section{2 - Expérience sur pin de Corse.}

Cette expérience a pour but de contròler l'effet d'une fumure (la mème que pour le pin sylvestre), de trois écartements de plantation (cfr. pin sylvestre) et de comparer deux travaux du sol (fraisage par bandes de $50 \mathrm{~cm}$ de largeur après sous-solage à $60 \mathrm{~cm}$ de profondeur suivant laxe des bandes d'une part et fraisage en plein à $10 \mathrm{~cm}$ de profondeur d'autre part), sur la croissance du pin de Corse.

\section{3 - Description des expériences.}

\section{1 - Matériel expérimental.}

311 - Lieu.

Les deux champs d'expérience sont situés à proximité l'un de l'autre, sur le plateau mosan de Mechelen-aan-de-Maas, au lieu-dit "Onder de Heide , à une altitude de $90 \mathrm{~m}$ environ. 
Le terrain présente une faible pente (inférieure à $1 \%$ ) de sorte que les expositions varient légèrement d'un champ à l'autre: NordOuest pour le pin de Corse, Sud-Est pour le pin sylvestre. La végétation comporte les plantes typiques d'une lande à bruyère.

$312-$ Sol.

312.1 - Roche-mère.

La roche-mère est une formation pléistocène d'origine alluviale (dépót de terrasse de la Meuse) recouverte par un dépót éolien. Elle présente une texture assez variable, mais principalement caractérisée par d'épaisses couches graveleuses dans lesquelles se retrouvent des débris de roches de toutes natures et de toutes formes, originaires du territoire d'érosion ardennais de la Meuse et de ses affluents.

On y reconnait facilement des signes d'altération glaciaire. Les vides sont comblés par des éléments sableux.

Cette formation atteint dans son ensemble plusieurs mètres d'épaisseur et est entrecoupée de couches à texture plus fine, voire même sans cailloux.

A l'endroit où sont situés les champs d'expérience, s'étend en surface une couche sableuse typique reposant entre 60 et $80 \mathrm{~cm}$ de profondeur sur une couche argilo-sableuse sans cailloux, peu perméable, déterminant la formation d'une nappe phréatique suspendue. La délimitation de ces dépóts est irrégulière par suite de phénomènes de cryoturbation.

\section{2 - Développement de profil.}

Le type de profil est un podzol à horizon $\mathrm{B}$ humique. Par suite de la présence d'une nappe suspendue temporaire à faible profondeur, il s'est formé un horizon ferrique extrêmement induré, séparé de l'horizon sous-jacent par un a iron pan $b$, mince couche ondulante (1 à $2 \mathrm{~mm}$ d'épaisseur) à haute concentration de sesquioxyde de fer.

I a faible perméabilité de l'horizon $B$ provoque la formation d'une nappe suspendue pédogène près de la surface $(40-45 \mathrm{~cm})$. Il en résulte un phénomène de sursaturation en périodes de pluies abondantes ou prolongées, provoquant l'inondation des sols. Cette économie en eau très particulière a provoqué des phénomènes de gley dans les horizons $\mathrm{A} 2$ et $\mathrm{Bh}$ bien que dans ce sol la réserve en eau soit négligeable.

\section{3 - Description du profil.}

\section{Données générales}

Profil $n^{\circ}$ 12. Feuille de la carte de Belgique, Zutendaal 26/6.

Description et échantillonnage: 9 octobre 1959.

Temps ensoleillé pendant une période prolongée de sécheresse. 


\section{Données concernant le profil (*)}

Série de sol: wzgdt - sol sableux, temporairement modérément humide, développé dans des dépóts de terrasse.

Substrat argilo-sableux à faible profondeur.

Développement de profil: podzol à horizon B humique et ferrique.

Végétation: bruyère à Calluna vulgaris, quelques Erica tetralix, Molinia coerulea assez abondante, tapis de mousse.

Roche-mère: dépót de terrasse de la Meuse, d’ảge pléistocène, caillouteux jusque dans l'horizon C (10-50 \%cailloux).

Situation physiographique: Haute Campine, plateau.

Altitude: $90 \mathrm{~m}$.

Relief : normal à subnormal; le profil est creusé stur une longue pente, très faible (inféricure à $1 \%$ ), face à la direction sud.

Drainage: - superficiel : très lent, stagnant;

- perméabilité: grande jusque dans l'horizon Bh humique, fortetement réduite dans l'horizon Bir, substrat très peu perméable;

- interne: très lent:

- classe de drainage: temporairement, modérément humide (Staunässe primaire et secondaire).

Erosion: érosion éolienne à craindre après disparition de la végétation.

Humidité: très sec jusque dans Thorizon Bh; horizons sous-jacents un peu moins secs.

Nappe phréatique: deux niveaux temporaires dans le profil (horizons Bir et D); eau phréatique permanente à très grande profondeur $(70-80 \mathrm{~m})$.

Pierrosité : environ $5 \%$ jusqu'ả $40 \mathrm{~cm}$ de profondeur; près de $25 \%$ au delà de $40 \mathrm{~cm}$ de profondeur.

Activité biologique: très faible.

Enracinement: les racines se cantonnent principalement dans l'horizon A1: dans Thorizon Bh, quelques cailloux sont entourés d'un fin chevelu.

Influence humaine: pas de perturbation de la morphologie du profil.

Remarque: les champs d'expérience font partie d'une grande bruyère fréquemment incendiée.

\section{Description du profil}

Horizon $\mathrm{A} 0$ : de 0 à $1 \mathrm{~cm}$; transition abrupte et régulière;

5 YR $3 / 2$ (sec également);

humus brut amorphe, grains minéraux peu abondants: tassé, feuilleté.

Horizon $\mathrm{A} 1$ : de 1 jusqu'à $4-6 \mathrm{~cm}$ de profondeur : transition abrupte et régulière;

10 XR $2 / 2$ avec grains de quartz délavés;

sable fin, faiblement humifère;

structure polyédrique angulaire très faible à granulaire;

dur, modérément résistant; environ $2 \%$ de cailloux.

Horizon A2: de 4-6 jusqu'à $30-35 \mathrm{~cm}$; transition abrupte et ondulée; 7,5 YR $5 / 2(\sec 7,5$ YR $7 / 2)$;

sable fin, peu humifère;

granulaire à très faiblement grumeleux;

très friable, pas résistant;

près de $5 \%$ de cailloux.

(*) Légende employé au Centre de Cartographie des sols, Gand. 
Remarque: les matières humiques sont réparties de façon irrégulière, se présentant sous forme de taches sombres dans un sable très clair; les radicelles se trouvent surtout sur les faces des cailloux.

Horizon Bh: de $30-35 \mathrm{~cm}$ jusqu'ì $31-40 \mathrm{~cm}$ : transition abrupte et irrégulière; 5 YR $3 / 2$ (sec également):

sable limoneux très humifère;

finement lamellé à granulaire:

friable, peu résistant:

environ $30 \%$ de cailloux.

Remarque: présence de coulées de l'horizon $\mathrm{Bh}$ jusque dans les horizons $\mathrm{C}$ et même $\mathrm{D}$.

Horizon Bir : de $31-40 \mathrm{~cm}$ jusqu'à $45-50 \mathrm{~cm}$ : transition abrupte et irrégulière ; 7,5 YR $4 / 4(\sec 10$ YR $6 /)$;

sable légèrement limoneux;

massif à grossièrement lamellé;

très dur, très résistant;

environ $20 \%$ de cailloux.

Remarque: entre 1'horizon Bh et Bir se trouve un e iron pan ? très dur et ondulant; présence de quelques bandes humiques (5 YR 3/3) d'une épaisseur de 1 à $2 \mathrm{~mm}$.

Horizon C $(\mathrm{B} 2 \mathrm{t})$ : de $45-50 \mathrm{~cm}$ à $70 \mathrm{~cm}$ : transition abrupte et irrégulière;

10 YR $5 / 1(\sec 10$ YR $6 / 4)$;

sable fin, sans matières humiques;

massif, dur et très résistant.

Remarque: présence de quelques taches $\mathrm{Br}$ dissout 7,5 YR 5/6: présence de quelques grandes taches de $\mathrm{Bh}$ :

environ $15 \%$ de cailloux.

Horizon D (B2t): supérieur à $70 \mathrm{~cm}$;

7,5 YR $5 / 8$ et 10 YR $6 / 3$;

sable argileux à argileux-sableux (sous forme de bandes); angulaire à lamellé dans le sable argileux, fortement angulaire dans les bandes argileuses;

dur (spécialement les taches brunes et les taches Bh);

environ $20 \%$ de cailloux.

Remarque: bariolé; alternance de bandes gris-pále et brun foncé, assez réguliềres et bien délimitées.

\section{4 - Résultats d'analyses et caractéristiques du profil pédologique.}

Les résultats d'analyses (voir tableau 1) confirment le caractère nettement sableux du profil.

La teneur en argile ne dépasse jamais $5 \%$ et descend jusqu'à $0,5 \%$ dans l'horizon A2. Elle s'élève à $15 \%$ dans les bandes argilosableuses et atteint $30 \%$ dans les bandes argileuses (colonne 0-2 $\mu$ ).

La fraction sableuse (colonne $50-2000 \mu$ ) représente 78 à $88 \%$ du total du profil; la majorité des grains relèvent de la classe 100 à $200 \mu$. 


\section{TABLEAU I}

Analyse et caractéristiques du profil pédologique.

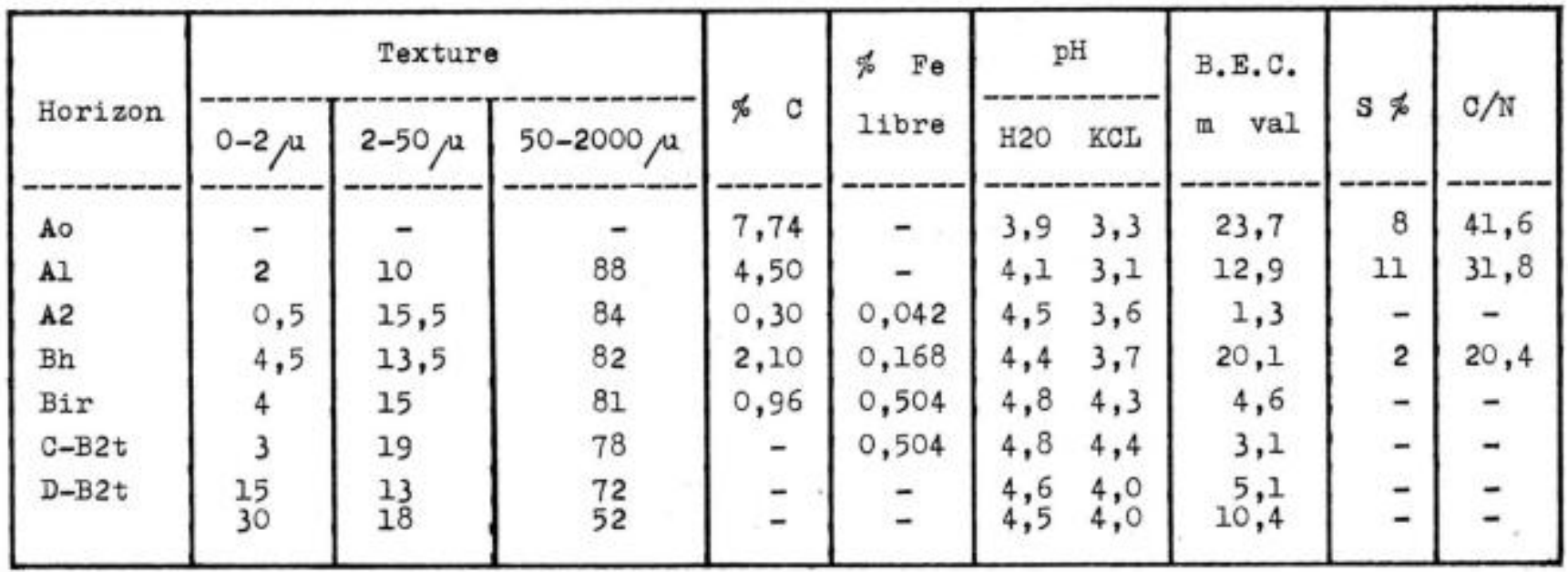


Le pourcentage de carbone $(\%$ C) décroit de $8 \%$ dans lhorizon A0 à $4,5 \%$ au niveau $\mathrm{A} 1$ pour être pratiquement négligeable dans l'horizon A2. L'horizon Bh, immédiatement sous-jacent, accuse une forte accumulation d'acide humique $(2,1 \%)$.

Les domnées fournies par l'analyse ne révèlent pas une accumulation prononcée de fer libre dans l'horizon $\mathrm{B}$ ferrique. La compacité de cette zone est vraisemblablement due à d'autres substances.

La réaction de l'entièreté du profil $(\mathrm{pH})$ est nettement acide malgré la faible augmentation de $\mathrm{pH}$ en profondeur ( 3,9 au niveau A0 à 4,8 dans l'horizon B).

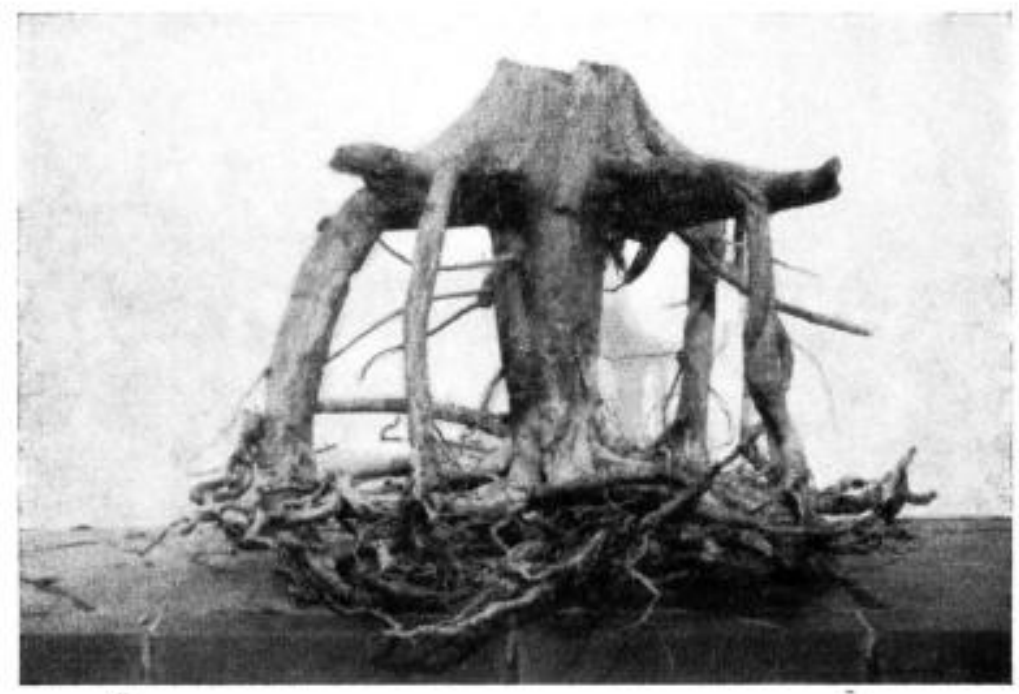

Рното 1.

Etalement des racines au niveau de la coupe ferrique.

La capacité d'échange en base est indiquée en milliéquivalents à la colonne B.E.C. ( $\alpha$ base exchange capacity $»$ ).

La capacité d'absorption (S \%), fonction directe de la teneur en matière organique du profil, est généralement assez élevée pour assurer une fixation satisfaisante des cations. La réserve naturelle en éléments minéraux est extrèmement faible: elle est insuffisante pour assurer l'alimentation des espèces résineuses, le taux de saturation ne dépassant pas $10 \%$.

L'économie en azote (rapport carbone-azote: $\mathrm{C} / \mathrm{N}$ ) s'avère défectueuse, le rapport $\mathrm{C} / \mathrm{N}$ s'exprimant par des valeurs très élevées.

Le profil est pauvre en minéraux altérables: on note la présence de feldspaths, la glauconie est absente. 
En résumé, il s'agit d'un sol sableux, très acide, de qualités physiques médiocres et très pauvre des points de vue chimique et biologique. Dans le cas présent, les défauts physiques inhérents à un profil du type poclzol, sont accentués par une économie en eau très défectueuse, due à la présence d'un $\alpha$ iren pan $»$ à faible profondeur. De plus, ce dernier est responsable de l'étalement des racines des plantes au niveau de la couche ferrique indurée, limitant ainsi l'exploitation des zones nutritives (voir photo $n^{\circ} 1$ ).

\section{3 - Choix des traitements.}

En ce qui concerne les amendements et le travail du sol, le choix des traitements est dicté par la nature mème du substrat expérimental qui vient d'être décrit. Le choix des écartements relève essentiellement de considérations économiques.

La mise en place des plants a été effectuée, en avril 1958 (par temps sec et chaud). suivant la méthode de plantation en fente.

Les pins sylvestres et les pins de Corse sont, les uns et les autres, ágés de 2 ans (S1 R1); les premiers sont originaires d'un peuplement de la région d'Aerschot, réputé pour sa forme et son rendement: les seconds proviennent du peuplement de Koekelaere, bien connu des forestiers.

\section{1 - Amendements.}

L'enrichissement chimique dont on souhaite contròler l'effet sur la croissance des pins sylvestres et des pins de Corse, a été réalisé en deux temps:

a) en automne 1957, 2 tonnes de marne et 1 tonne de scories de déphosphoration à l'ha, ont été épandues en plein avant le travail du sol.

b) au printemps 1959, soit un an après la plantation, $200 \mathrm{~kg}$ de chlorure de potasse $20 \%$ et $100 \mathrm{~kg}$ de nitrate d'ammoniaque $17 \%$ à l'ha, ont été appliqués en plein.

\section{2 - Trazail du sol.}

Dans l'essai sur pin sylvestre, le sol a été travaillé uniformément dans le courant de l'automne 1957: fraisage à $10 \mathrm{~cm}$ de profondeur sur des bandes de $50 \mathrm{~cm}$ de largeur après un sous-solage à $60 \mathrm{~cm}$ de profondeur dans l'axe des bandes. Les axes sont distants de $1 \mathrm{~m}$ les uns des autres de sorte que la bruyere est maintenue entre les bandes sur une largeur de $50 \mathrm{~cm}$ (voir photo $\mathrm{n}^{\circ} 2$ ).

Le fraisage ameublit le sol, mélange les horizons supérieurs et déracine la bruyère; le sous-solage brise l'horizon ferrique imper- 
méable décrit antérieurement et favorise le drainage en période de pluie prolongée (Hurgh, 1957; Van Goor, 1952).

Mais ce traitement du sol a le désavantage d'étre couteux (1850 F.B./ha), C'est pourquoi l'on a jugé intéressant de le comparer,

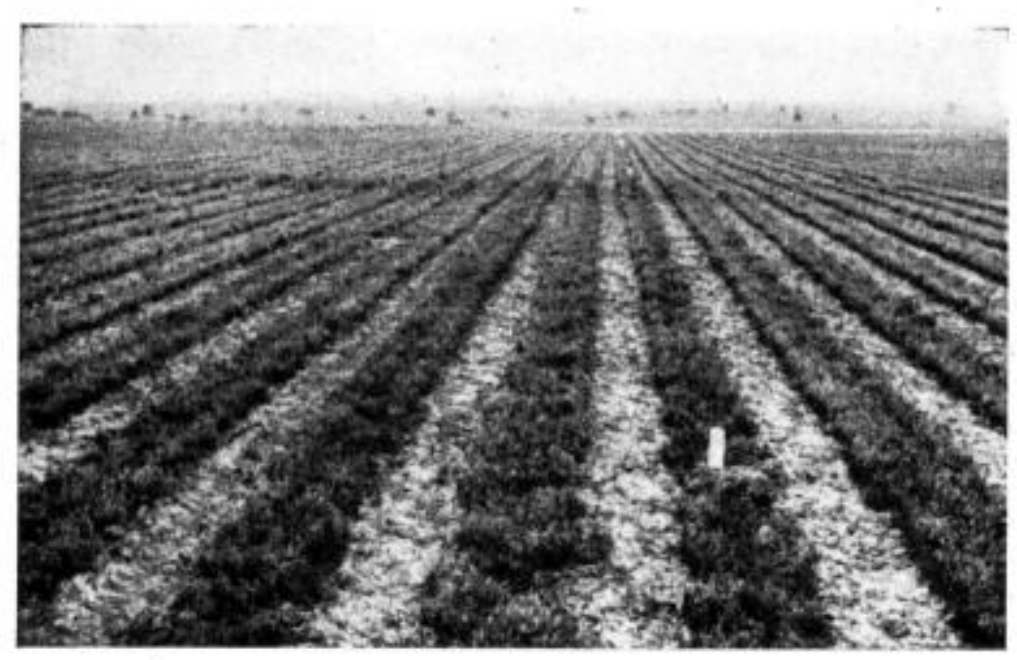

Pното 2.

Aspect général du champ fraisé par bandes.

dans le cas du pin de Corse, à une technique plus sommaire consistant simplement en un fraisage en plein a $10 \mathrm{~cm}$ de profondeur, dont le coût est réduit à 1275 F.B. ha.

\section{3 - Ecartements de plantation.}

L'écartement de plantation des pins sylvestres et de Corse est traditionnellement de $1 \mathrm{~m} \times 1 \mathrm{~m}$ (10000 plants à l'ha). II serait intéressant d'un point de vue économique, de pouvoir réduire le nombre de plants à l'ha à la moitić ou au quart. C'est pourquoi, tant pour le pin sylvestre que pour le pin de Corse, les écartements choisis dans nos essais sont de $1 \mathrm{~m} \times 1 \mathrm{~m}$, de $1 \mathrm{~m} \times 2 \mathrm{~m}$ et de $2 \mathrm{~m} \times 2 \mathrm{~m}$.

\section{2 - Plan des expériences.}

Dans les deux champs d'expérience, les parcelles sont de dimensions constantes $(25 \mathrm{~m} \times 25 \mathrm{~m})$ de sorte que le nombre initial d'arbres par parcelle est variable suivant l'écartement de plantation. 
Les doses étudiées pour chaque traitement sont symbolisées comme suit:

\begin{tabular}{|c|c|c|}
\hline Iraitecenta & Doses & Symboles \\
\hline Engrais (En) & $\begin{array}{l}\text { Absence d'engruls } \\
\text { Prósence d'engrais }\end{array}$ & $\begin{array}{l}c_{0} \\
e_{1}\end{array}$ \\
\hline Soua-solage (Ss) & $\begin{array}{l}\text { Absence de sous-solage } \\
\text { Préserce de eoug-solage }\end{array}$ & $\begin{array}{l}a a_{0} \\
a s_{1}\end{array}$ \\
\hline Ecartewent de plsntstion ( $\left.\mathrm{B}_{\mathrm{c}}\right)$ & 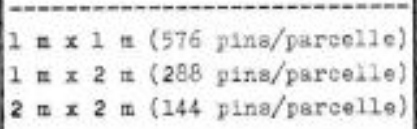 & $\begin{array}{l}11 \\
12 \\
22\end{array}$ \\
\hline
\end{tabular}

321 - Plan de l'expérience sur pin sylvestre.

L'expérience sur pin sylvestre dans laquelle on ne contróle que l'effet de l'engrais et des écartements de plantation, est conçue suivant un plan factoriel $2 \times 3$ en 3 blocs au hasard (Cox et CocHran, 1957); les 6 combinaisons de traitements auxquelles il donne lieu $\left(e_{0}-11, e_{0}-12, e_{0}-22, e_{1}-11, e_{1}-12, e_{1}-22\right)$, sont réparties aléatoirement dans chacun des 3 blocs (ou répétitions) stuivant le schéma donné dans le tableau II.

TABLEAU II

Plan dexpérience sur pin sylvestre.

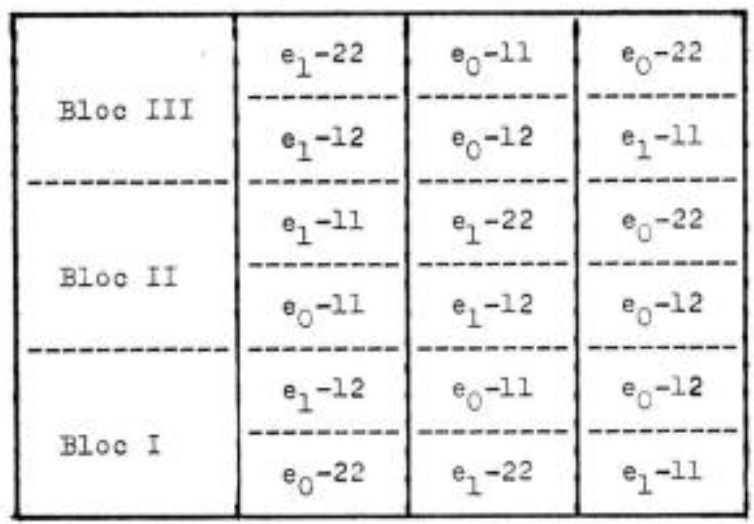

\section{2 - Plan de lexpérience sur pin de Corse.}

Dans l'expérience sur pin de Corse, où les trois traitements entrent en jeu, le plan adopté est un plan factoriel $(3 \times 2) \times 2$ arrangé en un "half-plaid square " $6 \times 6$ (Cox et Cochran, 1957) 
dans lequel on ne peut dégager une estimation précise de l'effet du sous-solage et par lequel on confond partiellement les interactions $\mathrm{E}_{n} \times \mathrm{S}_{\mathrm{s}}$ et $\mathrm{E}_{1} \times \mathrm{S}_{\mathrm{s}} \times \mathrm{Ec}$, les informations relatives étant respectivement de $8 / 9$ et de $5 / 9$ : ces interactions sont confondues avec les colonnes Ia, Ib, IIa, IIb, IIIa et IIIb du carré (square).

Ce dispositif expérimental dans lequel la moitié du champ d'essai subit un fraisage en plein sans sous-solage et l'autre moitié un fraisage en handes avec sous-solage, répond à des impératifs d'ordre technique: de tels travaux ne peuvent être exécutés pratiquement que sur de grandes surfaces, en tout état de cause supérieures à celle des parcelles.

Le plan de l'expérience sur pin de Corse est donné dans le tableau III.

TABLEAU III

Plan d'expérience sur pin de Corse.

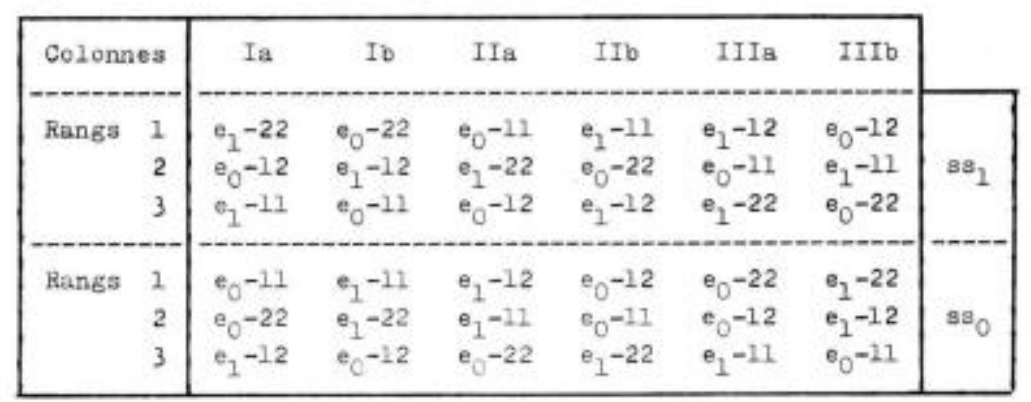

\section{4 - Analyse statistique des résultats expérimentaux.}

I a croissance individuelle des pins sylvestres et des pins de Corse est suivie de trois en trois ans; les plants morts en cours d'essai ont été remplacés de manière à ce que les écartements de plantation soient respectés, mais les plants de regarnissage n'entrent pas en ligne de compte dans nos calculs de rendements.

Les premières mesures ont été effectuées en juin et juillet 1962 , à savoir la longueur totale atteinte par chaque arbre en 1961 ainsi que la hauteur des pousses 1960 et 1961. Ceci nous a permis de calculer la hauteur totale par arbre en 1959 et 1960 .

\section{1 - Résultals de lexpérience sur pin sylvestre.}

Les distributions des différentes mesures individuelles par catégories de hauteurs sont généralement symétriques dans le cas du pin sylvestre; on admet qu'elles sont gaussiennes (tests graphiques après transformation en probits des fréquences cumulées $\%: \mathrm{cfr}$. Fisher et Yates, 1957, table IX). Ceci est en accord avec des résultats obtenus antérieurement au Centre de Bokrijk (NEF, 1959). 
D'autre part, on peut accorder le même poids aux moyennes de ces valeurs par parcelle (données dans les tableaux IV à VIII) bien qu'elles soient basées dans chaque bloc sur des nombres d'arbres allant du simple au double ou au quadruple d'une parcelle à l'autre suivant les écartements. En effet, le nombre minimum de pins sylvestres par parcelle étant élevé (théoriquement: 144, pratiquement: 137), on peut s'attendre à ce que la variabilité des mesures entre les arbres danz les parcelles soit homogène pour l'ensemble de l'essai. Ceci est vérifié à propos de la hauteur des pins en 1961 : à une exception près (parcelle $e_{1}-11$, bloc $\mathrm{I}$ ), les variances intraparcelles ne sont pas significativement différentes les unes des autres (test d'homogénéité des variances de Bartlett: $\chi^{2}=24,251$ avec 16 d.1.; cfr. Snedecor, 1956). Nous présentons dans le tableau IX, les variances intraparcelles $\left(\mathrm{s}^{2}\right)$ en question, ainsi que les nombres d'arbres vivants en $1961(\mathrm{~N})$, les hauteurs moyennes $\left(\bar{H}_{61}\right)$ et leurs limites fiduciaires à $95 \%$, à savoir $\bar{H}_{61}-$ t.s $\overline{\mathrm{H}}$ et $\bar{H}_{61}+t_{s} s^{\bar{H}}$ (les valeurs $t$ de StUdent sont données par FisHer et YATEs, 1957, table III), et les coefficients de variation

$$
\mathrm{CV} \%-\frac{100 \mathrm{~s}}{\overline{\mathrm{H}}_{61}} \text {. }
$$

Dans ces conditions, nous sommes en mesure d'effectuer, sur ces moyennes, des analyses de variance en vue d'un contrôle statistique des effets éventuels des traitements.

Les carrés moyens des analyses de variance effectuées pour chaque type de mesures, sont groupés dans le tableau X. Les carrés moyens relatifs aux effets des traitements sont testés par rapport aux carrés moyens de l'interaction blocs $\times$ traitements, compte tenu des nombres de degrés de liberté (d.1.) dont ils sont affectés (test F, efr. Fisher et Yates, 1957, table V). Les valeurs significatives aux seuils $5 \%, 1 \%$ ou $1 \%$ sont dotées respectivement d'un, de deux ou de trois astérisques.

A l'examen des analyses de variance du tableau $X$, on peut tirer les conclusions suivantes, valables dans les limites de notre essai:

1) L'effet de l'engrais se marque à partir de 1960, soit 2 ans après la plantation.

2) Les écartements n'ont aucun effet significatif sur la croissance du pin sylvestre, ce qui est normal vu le jeune âge de la plantation.

3) Il n'y a pas d'interaction engrais $X$ écartements.

Comme l'engrais seul a un effet sur la croissance des pins sylvestres, nous ne reprenons dans le tableau XI que les moyennes 
correspondant à ce facteur (voir aussi graphique 1). Parallèlement, nous donnons pour chaque type de mesures:

- les différences entre les moyennes obtenues avec et sans engrais, notées $\left(e_{1}-e_{0}\right)$;

- ces différences exprimées en pourcents de la moyenne obtenue sans engrais, notées $100\left(e_{1}-e_{0}\right) / e_{0}$; c'est une mesure de l'accroissement relatif de rendement dû̀ l'application d'engrais.

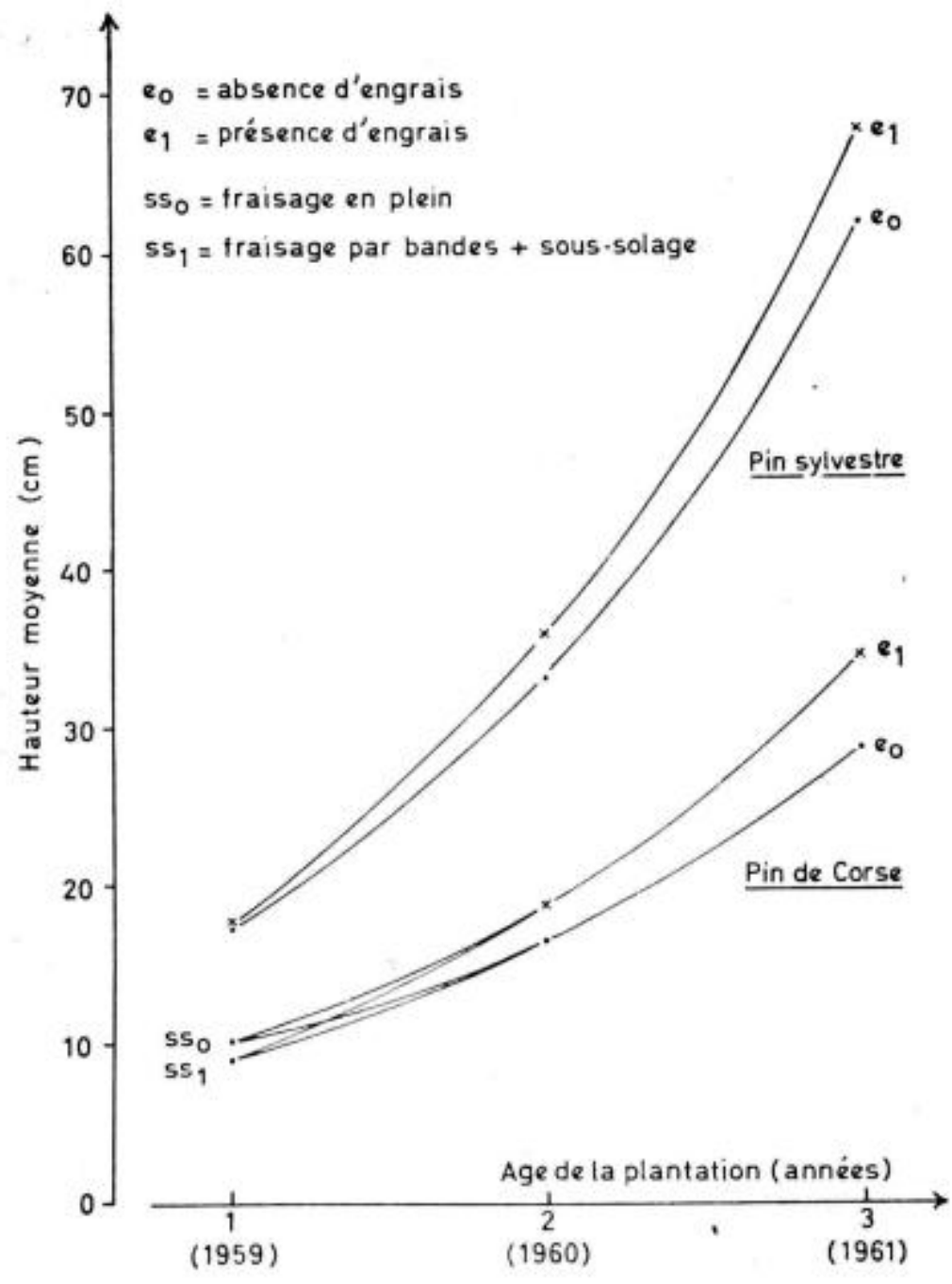

GRAPHIQUE 1

Croissance du pin sylvestre et du pin de Corse. 


\section{TABLEAU IV}

Hauteur moyenne $\bar{H}(\mathrm{~cm})$ des pins sylvestres en 1959 par parcelle, bloc et traitement.

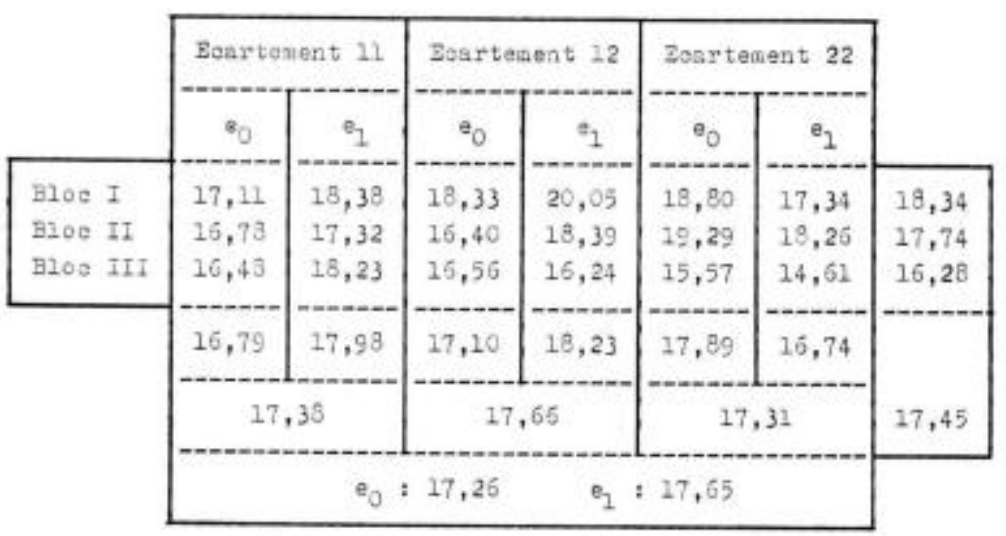

TABLEAU $V$

Longueur moyenne des pousses terminales $\overline{\mathrm{P}}(\mathrm{cm})$ des pins sylvestres en 1960 par parcelle, bloc et traitement.

\begin{tabular}{|c|c|c|c|c|c|c|c|}
\hline & \multicolumn{2}{|c|}{ Ecarteneat 11} & \multicolumn{2}{|c|}{ Beartevent 12} & \multicolumn{2}{|c|}{ Zaartersen: 22} & \\
\hline & $e_{0}$ & $e_{1}$ & $e_{0}$ & $e_{1}$ & ${ }^{6} \mathrm{O}$ & $e_{1}$ & \\
\hline 31001 & 16,36 & 17,16 & 15,70 & 18,01 & $\underline{15,82}$ & 19,64 & $\underline{17,12}$ \\
\hline $\mathrm{BLO0} \mathrm{II}$ & $\underline{15,94}$ & 20,30 & 15,42 & 19,04 & 16,50 & 19,60 & 17,48 \\
\hline \multirow[t]{3}{*}{8100 III } & 15,27 & 17,14 & $\underline{15,83}$ & 18,70 & $\underline{15,80}$ & $\underline{17,33}$ & 16,68 \\
\hline & $\underline{15,86}$ & 18,20 & 15,65 & 18,25 & 16,07 & 18,52 & \multirow{2}{*}{17,09} \\
\hline & & & & & &, 30 & \\
\hline & & ${ }^{e} \mathrm{O}$ & 15,86 & & 18,32 & & \\
\hline
\end{tabular}




\section{TABLEAU VI}

Hauteur moyenne $\overline{\mathrm{H}}(\mathrm{cm})$ des pins sylvestres en 1960 par jarcelle, bloc et traitement.

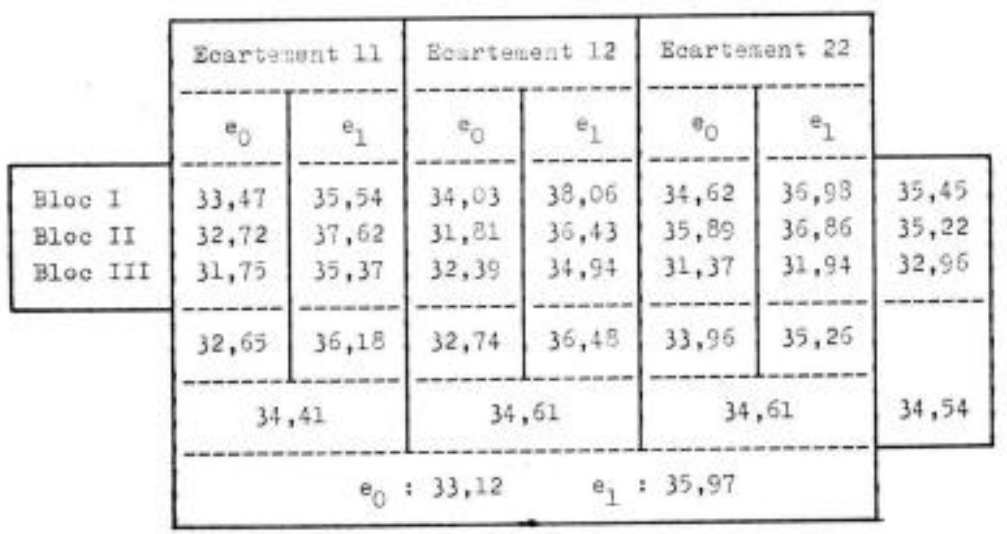

\section{TABLEAU VII}

Longueur moyenne des pousses terminales $\overline{\mathrm{P}}(\mathrm{cm})$ des pins sylvestres en 1961 par parcelle, bloc et traitement.

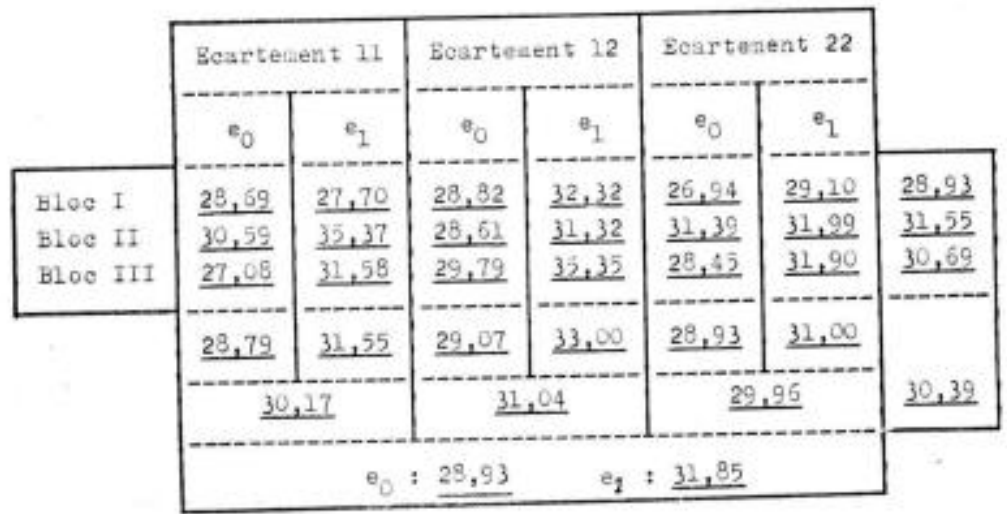




\section{TABLEAU VIII}

Hauteur moyenne $\overline{\mathrm{H}}(\mathrm{cm})$ des pins sylvestres en 1961 par parcelle, bloc et traitement.

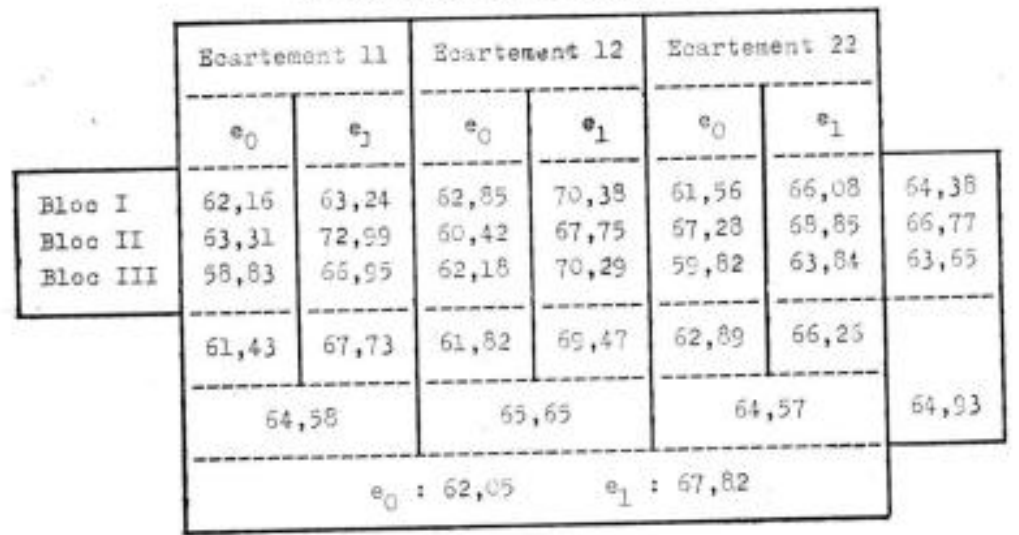

\section{TABLEAU IX}

Nombre de pins sylvestres par parcelle $(\mathrm{N})$, variances intra-parcelles $\left(s^{2}\right)$, hauteurs moyennes en $1961\left(\overline{\mathrm{H}}_{61}\right)$ encadrées de leurs limites fiduciaires $95 \%$ et coefficients de variation $\mathrm{CV} \%$.

\begin{tabular}{|c|c|c|c|c|c|c|c|}
\hline & & $\mathbb{E}$ & $\begin{array}{c}s^{2} \\
\left(\cos ^{2}\right)\end{array}$ & $\begin{array}{c}\bar{H}-t, z \cdot \bar{H} \\
(0 x)\end{array}$ & $\begin{array}{l}\overline{\mathrm{H}}_{61} \\
(\mathrm{sin})\end{array}$ & $\begin{array}{c}\vec{H}+t, s, \vec{H} \\
\langle o n\rangle\end{array}$ & $\mathrm{CV} \&$ \\
\hline \multirow{3}{*}{$0_{0}=11$} & bloe I & 549 & 314,7851 & 60,68 & 62,16 & 63,64 & 28,5 \\
\hline & bloc II & 553 & 352,6327 & 61,74 & 63,31 & 64,88 & 29,7 \\
\hline & blob III & 550 & 320,8426 & 57,33 & 58,93 & 50,33 & 30,4 \\
\hline \multirow{3}{*}{$g_{1}-11$} & bloc I & 549 & 403,2823 & 61,36 & 63,24 & 64,92 & 31,8 \\
\hline & bloc II & 569 & 349,1879 & 71,45 & 72,29 & 74.53 & 25,6 \\
\hline & bloo III & 569 & 296,7655 & 65,53 & 66,95 & 68,37 & 25,7 \\
\hline \multirow{3}{*}{$e_{0}-12$} & bloc I & 274 & 318,1222 & 60,74 & 62,85 & 64,76 & 28,4 \\
\hline & bloc II & 284 & 307,2561 & 36,38 & 60,42 & 62,46 & 29,0 \\
\hline & bloc III & 276 & 277,4559 & 60,21 & 62,18 & 64,15 & 26,8 \\
\hline \multirow[t]{3}{*}{$c_{1}-12$} & blod I & 282 & 278,2732 & 68,43 & 70,38 & 72,33 & 23,7 \\
\hline & bloo II & 278 & 276.5764 & 65,79 & 67,75 & 69,71 & 24,5 \\
\hline & blod 111 & 287 & 306,7816 & 65,26 & 70,29 & 72,32 & 24,9 \\
\hline \multirow{3}{*}{$e_{0}-22$} & bloe I & 144 & 204,1507 & 59,20 & 52,56 & 63,92 & 23,2 \\
\hline & bloo II & 143 & 330,5305 & 64,27 & 57,28 & 70,29 & 27,0 \\
\hline & bloo III & 138 & 271,4564 & 57,04 & 59,92 & 62,50 & 27.5 \\
\hline \multirow{3}{*}{$e_{1}-22$} & bloc I & 140 & 301,3931 & 63,17 & 66,08 & 58,99 & 26,3 \\
\hline & bloc II & 137 & 300,6403 & 55,92 & 68,85 & 71,78 & 25,2 \\
\hline & bloo III & 137 & 279,6732 & 61,01 & 63,84 & 66,57 & 26,2 \\
\hline
\end{tabular}




\section{TABLEAU $\mathrm{X}$}

Carrés moyens (C.M.) des analyses de variance par type de mesures (les valeurs significatives aux seuils $5 \%, 1 \%$ ou $1 \%$ sont dotées respectivement d'un, de deux ou de trois astérisques).

\begin{tabular}{|c|c|c|c|c|c|c|}
\hline & a.1. & $\begin{array}{c}\text { Hauteur moyenne } \\
1959\end{array}$ & $\begin{array}{c}\text { Pousce terni- } \\
\text { nale moyenne } \\
1950\end{array}$ & $\begin{array}{c}\text { Hauteur moyenne } \\
1960\end{array}$ & $\begin{array}{c}\text { Pousse termi- } \\
\text { nale moyenne } \\
1961\end{array}$ & $\begin{array}{c}\text { Hauteur moyenne } \\
1961\end{array}$ \\
\hline Bloos & 2 & 6,6969 & 0,9704 & 11,3673 & 10,6845 & 15,9354 \\
\hline Traitementa & 5 & 1,2843 & $5,5620^{* \pi}$ & $8,4581^{\star}$ & $\overline{8,9724}$ & $33,7908^{*}$ \\
\hline Engraia & 2 & 0,6805 & $27,3553^{* k *}$ & $36,6653^{\mathbf{k x}}$ & $38,3396^{\mathrm{k}}$ & $149,9912^{k \times x}$ \\
\hline Boartements & 1 & 0,2051 & $\underline{0,2018}$ & 0,0786 & $\underline{1,9459}$ & 2,2864 \\
\hline Engra1s $x$ écartementa & 1 & 2,6654 & $\overline{0,0256}$ & 2,7338 & $\underline{1,3153}$ & 7,1950 \\
\hline Bloca $\times$ Tmta & 6 & 1,2433 & 0,8759 & 1,6823 & $\underline{3,9453}$ & 7,8065 \\
\hline
\end{tabular}




\section{TABLEAU XI}

Données relatives à l'effet de l'engrais

sur la croissance du pin sylvestre.

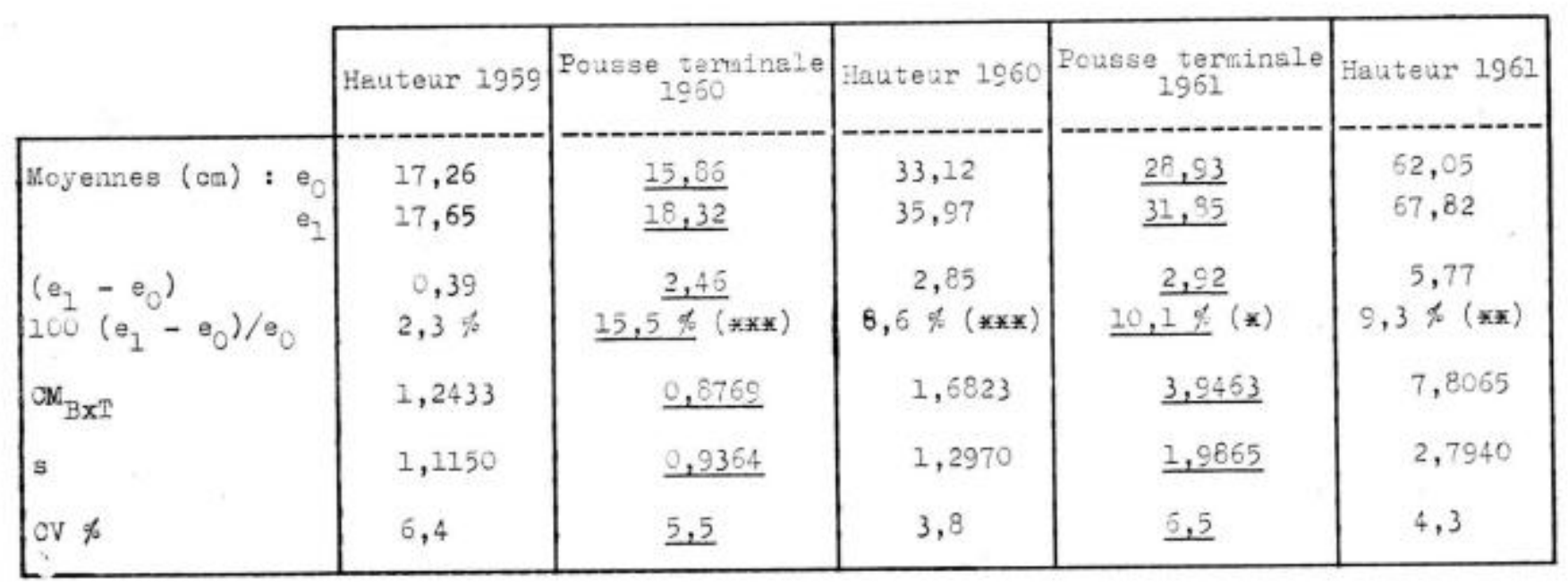


Les pourcentages sont dotés d'un, de deux ou de trois astérisques, selon que le carré moyen correspondant à l'effet des engrais est significatif aux seuils $5 \%, 1 \%$ ou $1 \%$ (cfr, tableau X). Afin de justifier ces astérisques, nous rappelons dans le tableau XI les valeurs des carrés moyens de l'interaction blocs $\times$ traitements (СМвт), par rapport auxquelles sont testés les effets des engrais. Enfin, nous donnons la racine carrée de ces carrés moyens, c'està-dire l'écart étalon de l'erreur (s) et le coefficient de variation $\mathrm{CV} \%=(100 . \mathrm{s}) / \overline{\mathrm{H}}$ ou $(100 . \mathrm{s}) / \overline{\mathrm{P}}$ selon le cas. On observe que ces coefficients sont du mème ordre de grandeur pour tous les types de mesures.

Enfin, il est également intéressant de comparer les pourcentages observés de pins sylvestres qui atteignent et dépassent une hauteur donnée à un âge donné. Ces pourcentages figurent dans le tableau XII. On peut observer par exemple, que $16,2 \%$ des plants n'ayant pas reçu d'engrais ont une hauteur en 1961 égale ou supérieure à $80 \mathrm{~cm}$, alors que dans le cas des arbres ayant recu de l'engrais, ce pourcentage est de $27,1 \%$.

\section{TABLEAU XII}

Pourcentages de pins sylvestres

ayant atteint ou dépassé en 1961 une hauteur donnée $\mathrm{X}_{1}$.

\begin{tabular}{|c|c|c|}
\cline { 2 - 3 } \multicolumn{1}{c|}{} & $\begin{array}{c}\text { Bn absence } \\
\text { d'engrais } \\
\mathrm{X}_{1}=10 \mathrm{~cm}\end{array}$ & $\begin{array}{c}\text { En présence } \\
\text { d'engrais } \\
\mathbf{e}_{1}\end{array}$ \\
\hline 20 & $100,0 \%$ & $100,0 \%$ \\
30 & 98,9 & 99,6 \\
40 & 96,1 & 97,6 \\
50 & 89,1 & 92,9 \\
60 & 74,8 & 84,2 \\
70 & 55,6 & 68,8 \\
80 & 34,0 & 48,6 \\
90 & 16,2 & 27,1 \\
100 & 5,3 & 11,3 \\
210 & 1,2 & 3,7 \\
120 & 0,3 & 0,9 \\
& & 0,1 \\
\hline
\end{tabular}

42 - Résultats de lexpérience sur pin de Corse.

La distribution des hauteurs totales en 1961 n'est généralement pas symétrique: il y a surabondance de plants dans les classes de 
fréquences de 0 à $9,9 \mathrm{~cm}$ et de 10 à $19,9 \mathrm{~cm}$. Ceci s'explique par le fait que la plupart de ces plants ont végété depuis le moment de leur plantation: ils n'ont pas eu de pousse terminale en 1960 et en 1961 (dégáts dus aux coqs de bruyère). En 1961, 22.3 \% des pins vivants sont dans ce cas: le tableau XIII montre comment ils se répartissent en fonction des traitements. Ce tableau sera commenté plus loin.

Les hauteurs moyennes $(\overline{\mathrm{H}})$ des pins de Corse en 1959, 1960 et 1961, et les longueurs moyennes des pousses terminales $(\overline{\mathrm{P}}) 1960$ et 1961 par traitements et combinaisons de traitements figurent dans les tableaux XIV à XVIII.

L'analyse statistique porte sur les réstultats moyens par parcelle. On peut accorder le même poids à ces moyennes sur la base des arguments déjả développés pour l'étude des pins sylvestres.

Les carrés moyens des analyses de variance des hauteurs annuelles moyennes des pins de Corse et des longueurs moyennes des pousses terminales sont donnés dans le tableau XIX.

L'estimation de l'effet du sous-solage est imprécise: en se reportant au plan de l'essai, on conçoit que l'effet du sous-solage est confondu dans une mesure qu'on ne peut évaluer, avec l'effet des conditions ambiantes des deux moitiés du champ d'essai, réservées entièrement l'une au sous-solage, l'autre au fraisage.

Les interactions engrais $X$ sous-solage et écartements $X$ engrais $X$ sous-solage sont confondues avec les colonnes dans une mesure que l'on peut chiffrer à, respectivement, $8 / 9$ et $5 / 9$ de l'information. Leurs carrés moyens sont de ce fait corrigés (YATEs, 1937) et leurs degrés de liberté (d.1.) correspondants sont symboliquement repérés du signe "prime ".

A l'examen du tableau XIX, on observe qu'en 1959 le sous-solage a un effet significatif sur la hauteur des pins de Corse et quà partir de 1960, setul l'engrais agit significativement sur la croissance. Aucune des interactions n'est jamais significative.

Ces conclusions sont commentées ci-après:

\section{1) Engrais.}

L'engrais a un effet bénéfique sur la croissance dès 1960. I1 se dégage du tableau $\mathrm{XX}$ et du graphique 1 que l'action favorable de l'engrais s'affirme d'année en année, qu'elle soit exprimée en valeurs brutes $\left(e_{1}-e_{0}\right)$ ou en accroissements relatifs de rendements $\left[100\left(e_{1}-e_{0}\right) / e_{0}\right]$. On constate enfin que la reprise des pins de Corse est meilleure en présence d'engrais (cfr. tableaux XIII et $\mathrm{XX)}$.

\section{2) Travail du sol.}

Le tableau des moyennes ci-après met en évidence l'effet du travail du sol sur le comportement des pins de Corse au cours du 
temps. Rappelons qu'il est indissociable de l'effet du milieu ambiant dans les deux moitiés du champ d'expérience.

\begin{tabular}{|c|c|c|c|c|c|c|}
\hline & 苗 59 & $\overline{\mathbf{F}} 60$ & 而 60 & $\overline{\mathrm{P}}$ G1 & A 61 & $\begin{array}{l}\text { Pouroentaso da } \\
\text { planta végetant } \\
\text { en } 1961\end{array}$ \\
\hline $\boldsymbol{5 s}_{0}$ & 10,22 & 7,85 & 18,07 & 14,73 & 32,90 & 25,8 \& \\
\hline$s s_{1}$ & 9,05 & 3,31 & 17,36 & 12,90 & 30,26 & $17,9 \%$ \\
\hline
\end{tabular}

On observe que, d'une manière générale. la croissance est en moyenne meilleure dans la zone du champ fraisée complètement que dans la zone fraisée par bandes et sous-solée. La différence n'est cependant significative qu'en 1959 (seuil 5 \%). Par contre, la reprise est sensiblement meilleure dans la zone sotus-solée à en juger par le pourcentage de plants qui végètent en 1961 (plants sans pousses terminales 1960 et 1961). Notons ici que le pourcentage de plants morts en cours d'essai est du même ordre de grandeur quel que soit le traitement (en moyenne $5 \%$ ).

\section{3) Ecartements.}

Les écartements n'ont aucun effet significatif sur la croissance des pins de Corse, ce qui est normal vu le jeune âge de la plantation,

\section{5 - Interprétation biologique des résultats et conclusions.}

L'interprétation des premiers résultats des deux expériences décrites ici, l'une sur pin sylvestre, l'autre sur pin de Corse, ne conduit à des conclusions que dans des limites de temps très étroites: elles reposent exclusivement sur les observations faites au cours des trois premières années d'existence des plantations.

Dans ce cadre, nous analysons ci-après, successivement, les effets des trois traitements étudiés, sur la croissance en hauteur des arbres.

\section{1 - Engrais.}

Le sol campinois dans lequel sont installées les plantations expérimentales, est très acide et, à tous points de vue, d'une très grande pauvreté. C'est pourquoi une fumure équilibrée s'imposait. Selon plusieurs auteurs (Duchaufour et Guinaudeau, 1957; Bruning, 1961; Franz, 1956), un tel amendement doit comprendre au moins les quatre éléments suivants: $\mathrm{Ca}, \mathrm{P}, \mathrm{N}$ et $\mathrm{K}$. 


\section{TABLEAU XIII}

Pourcentages de pins de Corse ayant végété depuis le moment de leur plantation (absence de pousse terminale en 1960 et en 1961) et, notés entre parenthèses, nombres totaux de plants vivants en 1961 .

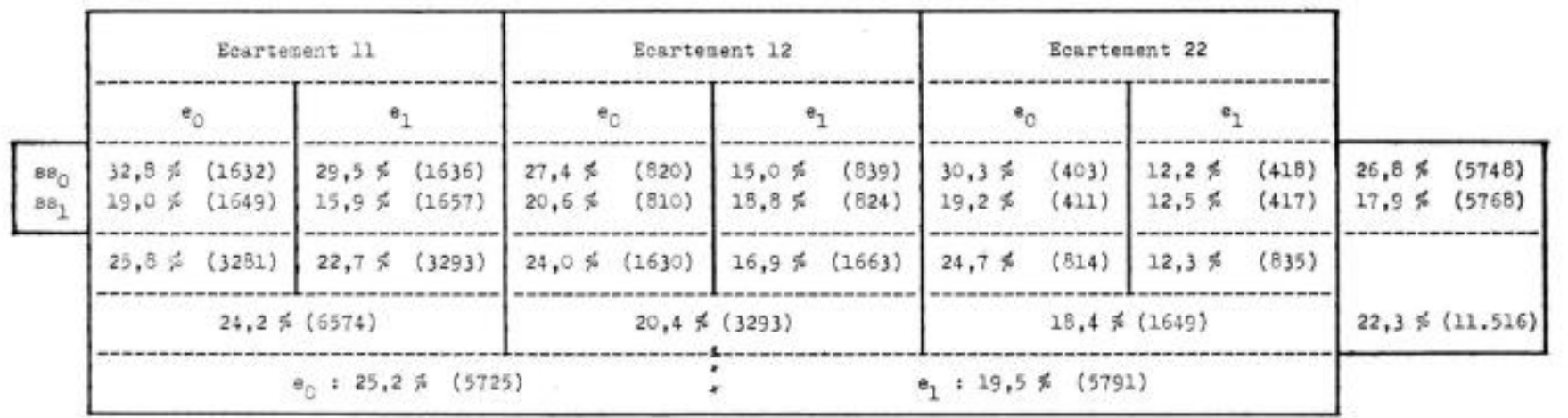

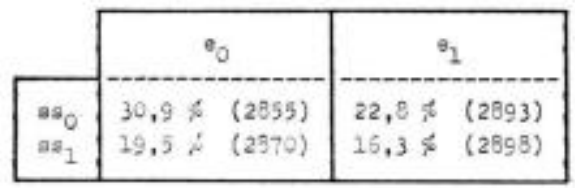

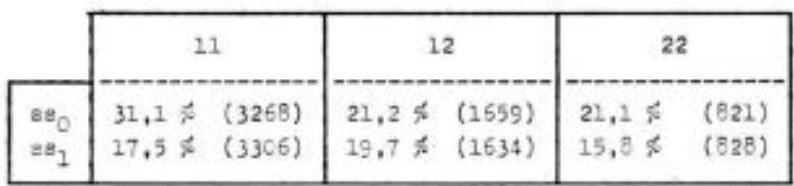


TABLEAU XIV

Hauteur moyenne $\overline{\mathrm{H}}(\mathrm{cm})$ des pins de Corse en 1959 par traitements et combinaisons de traitements.
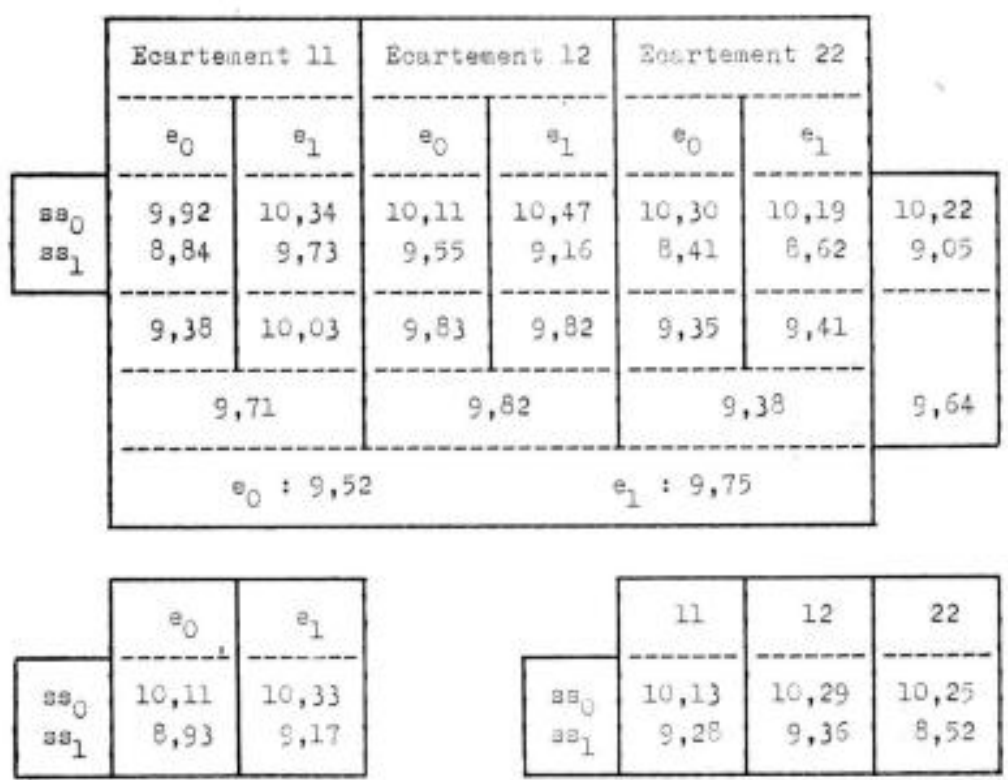

TABLEAU XV

Longueur moyente des pousses terminales $\overline{\mathrm{P}}(\mathrm{cm})$ des pins de Corse en 1960 par traitements et combinaisons de traitements.
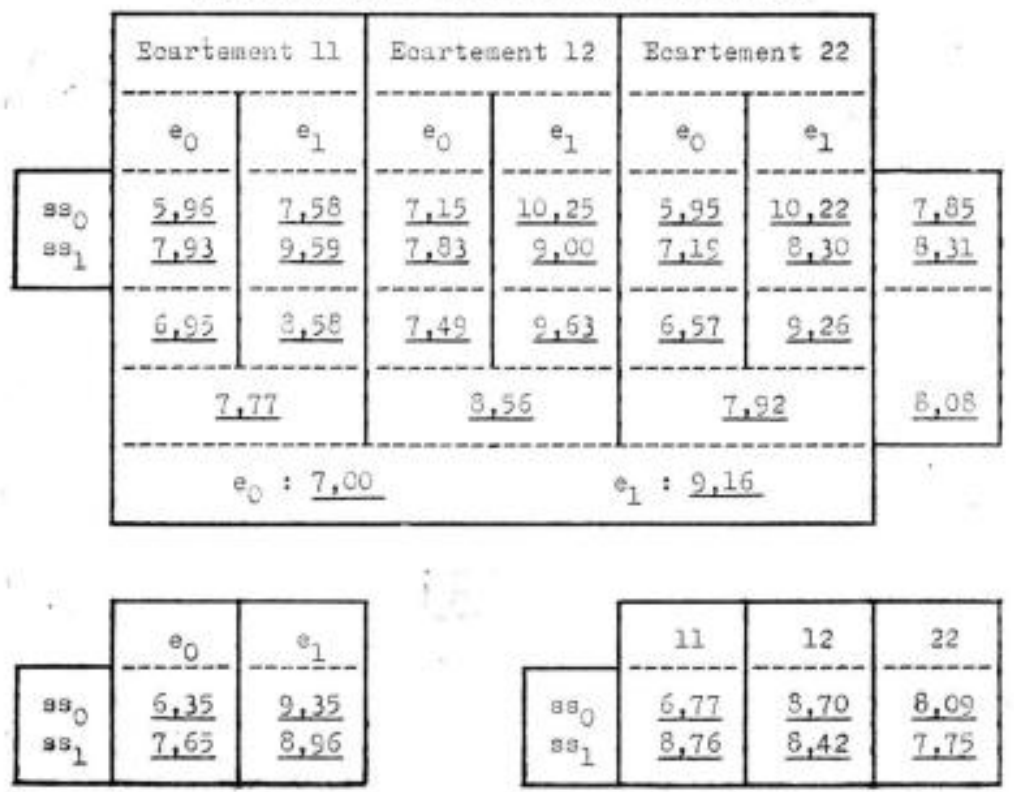
TABLEAU XVI

Hauteur moyenne $\mathrm{H}(\mathrm{cm})$ des pins de Corse en 1960 par traitements et combinaisons de traitements.
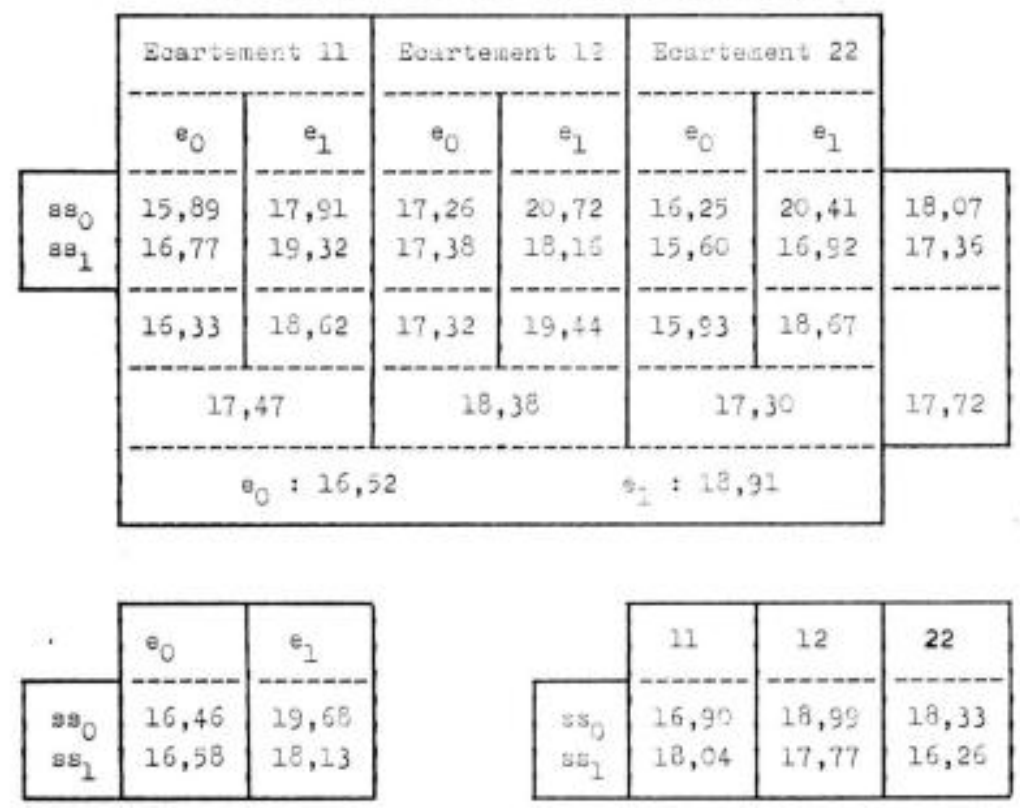

\section{TABLEAU XVII}

Longueur moyenne des pousses terminales $\overline{\mathbf{P}}(\mathrm{cm})$ des pins de Corse en 1961 par traitements et combinaisons de traitements.
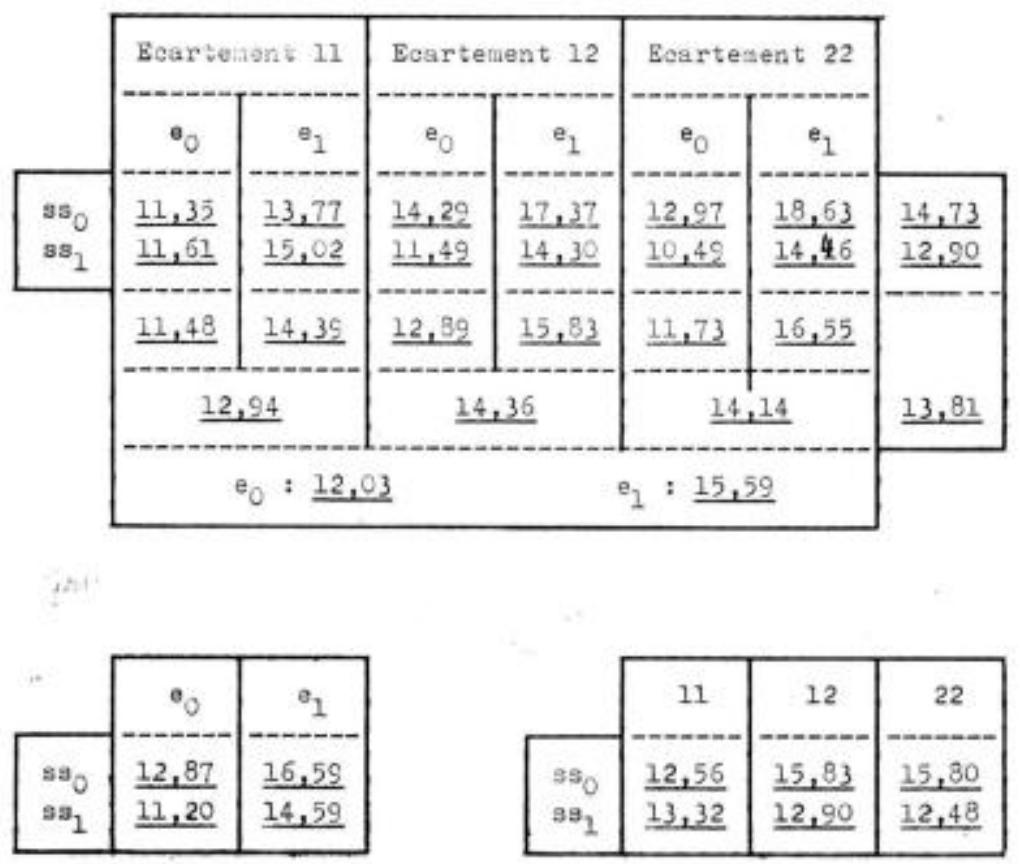
C'est ce qu'a réalisé le promoteur des expériences, F. Dufrane, par l'apport

1) de chaux et de phosphore enfouis dans le sol, sous une forme lentement soluble (marne, scories), en doses telles que l'on puisse en attendre un effet progressif et durable,

2) d'azote et de potasse appliqués en surface un an après la plantation, sous une forme rapidement soluble et de ce fait à faibles doses,

\section{TABLEAU XVIII}

Hauteur moyenne $\overline{\mathrm{H}}(\mathrm{cm})$ des pins de Corse en 1961 par traitements et combinaisons de traitements.
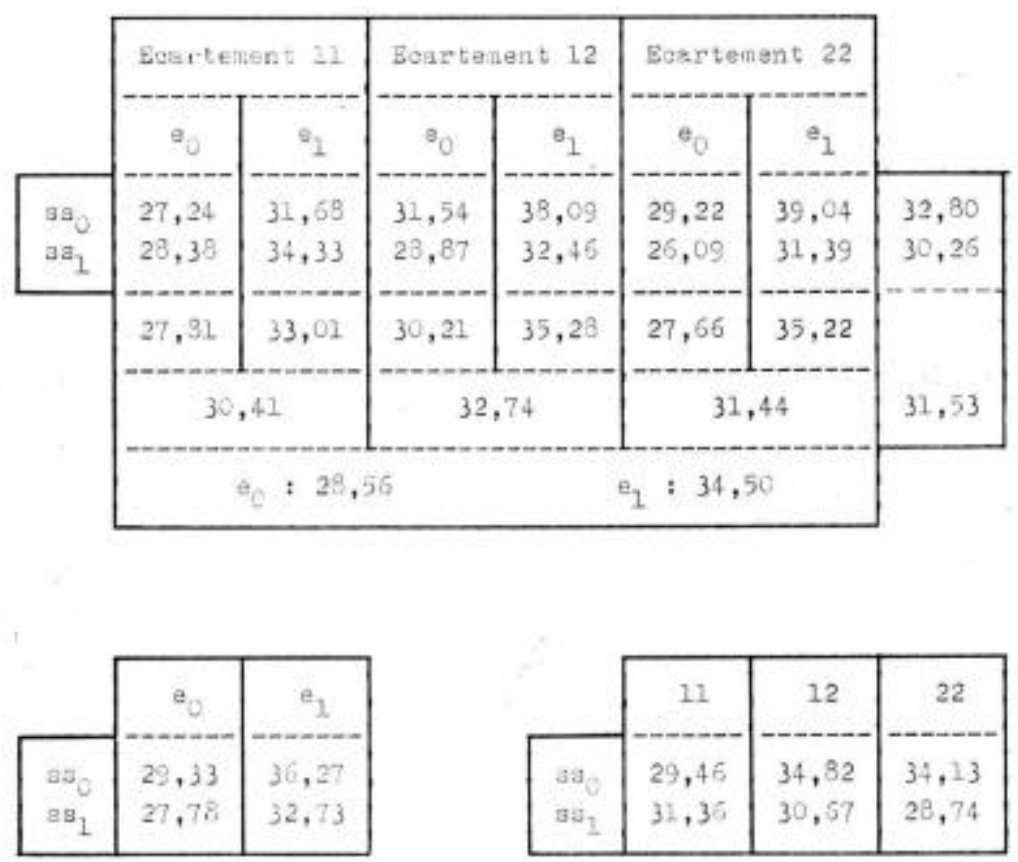

Une fumure de ce genre doit en principe favoriser la reprise des plants, augmenter la production dans l'immédiat, agir rapidement sur la flore et la faune de la biocénose et améliorer la fertilité du sol à longue échéance (Duchaufour, 1958; Ronde, 1960).

Dans les limites de nos essais, on peut constater que, de fait, la croissance des pins est rapidement favorisée par lapport d'engrais: les effets s'en font sentir dès la deuxième année (cfr, graphique 1). Bien que ceci soit une manifestation d'une reprise plus 


\section{TABLEAU XIX}

Carrés moyens (C.M.) des analyses de variance par type de mesures (les valeurs significatives aux seuils $5 \%, 1 \%$ ou $1 \% / 6$ sont dotées res. pectivement d'un, de deux ou de trois astérisques).

\begin{tabular}{|c|c|c|c|c|c|c|}
\hline & a.1. & $\begin{array}{c}\text { Hauteur moyenne } \\
1959\end{array}$ & $\begin{array}{l}\text { Pousse termina- } \\
\text { le moyenne } 1960\end{array}$ & $\begin{array}{c}\text { Hauteur moyenne } \\
1960\end{array}$ & $\begin{array}{l}\text { Pousse termina- } \\
\text { le moyenne } 13651\end{array}$ & $\begin{array}{c}\text { Hauteur moyenne } \\
1961\end{array}$ \\
\hline Atangs & 5 & 3,5357 & 7,5269 & 12,7223 & 26,4236 & 74,5474 \\
\hline Sous-aolage & 1 & $12,3201^{*}$ & 1,8632 & 4,6010 & 30,2133 & 58,3950 \\
\hline Erreur (1) & 4 & I, 3396 & 9,9429 & 14,7527 & 25,4762 & 78,5855 \\
\hline Colonnes & 5 & 0,5589 & 1,2612 & 1,8755 & 4,4905 & 11,6850 \\
\hline Engra1s & 1 & 0,4761 & $41,7952^{* * * \pi}$ & $51,1940^{\text {**: }}$ & 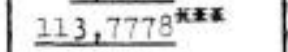 & $317,6118^{x \neq x=}$ \\
\hline Bcartements & 2 & 0,6305 & $\underline{2,1220}$ & 4,0653 & 7,0468 & 16,4108 \\
\hline Bo $x \mathrm{En}$ & 2 & 0,4039 & 0,8326 & 0,3085 & 3,5697 & 5,9014 \\
\hline Ec $\mathrm{x} \mathrm{Ss}$ & 2 & 0,7068 & $\underline{5,3155}$ & 8,3142 & 15,1944 & 45,6564 \\
\hline En $\times \mathrm{Ss}$ & $I^{\prime}$ & 0,0004 & $\underline{6,3589}$ & 6,2534 & 0,2401 & 8,9501 \\
\hline Bo $x \operatorname{Bn} \times \mathrm{Sa}$ & $2^{\prime}$ & 0,3355 & $\underline{1,9548}$ & 2,6970 & $\underline{1,3448}$ & 7,3572 \\
\hline Brreur (2) & 15 & 0,8996 & 1,6333 & 4,1267 & 4,5554 & 15,3930 \\
\hline
\end{tabular}


276 ESSAI D'AMÉLIORATION DES RENDEMENTS DU PIN SYLVESTRE




vigoureuse des plants en présence d'engrais, le taux de mortalité résultant de la transplantation est cependant à peine lié au traitement: le pourcentage d'arbres morts ou moribonds après trois ans d'essai est de $2.5 \%$ en présence d'engrais contre $3,7 \%$ en absence d'engrais dans le cas des pins sylvestres, et respectivement de 4.2 et de $5,3 \%$ dans le cas des pins de Corse. D'autre part, nous ne nous sommes pas préoccupés jusqu'ici de l'évolution de la flore et de la faune. Enfin, rien ne permet d'augurer de l'amélioration de fertilité du sol à longue échéance.

Il est également intéressant de noter que l'accroissement relatif de rendement dû à la fumure $\left\lceil 100\left(e_{1}-e_{0}\right) / e_{0}\right\rceil$ s'affirme d'année en année davantage dans le cas du pin de Corse que dans celui du pin sylvestre. En effet, il ressort des tableaux XI et XX que

1) la hauteur initiale moyenne des pins de Corse vaut environ la moitié de celle des pins sylvestres (en 1959, en absence d'engrais, respectivement 9,52 et $17,26 \mathrm{~cm}$ ).

2) l'accroissement relatif de rendement est du méme ordre de grandeur pour les deux essences en 1959 (2,4 et 2,3\%).

3) cet accroissement atteint en 1961, pour le pin de Corse, une valeur à peu près double de celle obtenue pour le pin sylvestre (respectivement 20,8 et $9,3 \%$ ).

Cette réaction plus accusée du pin de Corse en réponse à l'apport d'engrais est peut-être à mettre en parallèle avec le jaunissement des aiguilles qui, selon nos observations, est plus répandu dans son cas que dans celui des pins sylvestres. Cependant, nous ne pouvons conclure trop hâtivement, sur la base de nos expériences, que le pin de Corse est un utilisateur d'engrais plus rentable que le pin sylvestre. En effet, ces deux essences sont installées dans des champs d'essai distincts, bien que voisins, et de ce fait, on risquerait d'attribuer aux espèces ce qui n'est peut-être dû qu'aux conditions particulières des milieux. Mais si la supériorité du pin de Corse en la matière se confirmait, ceci ne ferait que renforcer d'autres qualités de croissance de cette essence qui ont justifié son introduction en Campine (Bodeux, 1949; Colpaert, 1937; Van Migroet et JansSENS, 1956).

Enfin, notons encore que nous avons observé, au cours de la première année de croissance, les manifestations d'une carence en azote dans nos deux champs d'expérience. Ceci est dû au fait que le sol. pauvre en cet élément, n'a été amendé de ce point de vue qu'un an après la plantation. Des constatations analogues ont été enregistrées également dans plusieurs essais forestiers à l'étranger (Duchaufour, 1957; Leyton, 1954; Van Goor, 1956). II eut donc été préférable d'apporter l'azote et la potasse peu de temps avant la plantation et de les enfouir par un simple hersage. 
En conclusion, on peut affirmer que la fumure équilibrée (Ca, P, $\mathrm{N}$ et $\mathrm{K}$ ) appliquée aux doses et de la manière décrites plus haut, a un effet positif très net sur la croissance du pin sylvestre et du pin de Corse dans le jeune âge et dans les conditions de nos expériences. Ces observations sont parallèles aux résultats obtenus dans les Landes dans des essais de fertilisation minérale sur pin maritime (Guinaudeau et al., 1963). Si l'on se réfère aux essais d'autres auteurs dans ce domaine (Duchaufour et Bonnead, 1960; Galoux, 1954), on peut espérer que cet avantage acquis dès le départ, aura des répercussions profitables sur la production finale.

\section{2 - Tratail du sol.}

Dans les landes campinoises, le travail du sol consiste généralement en un labour en plein avec enfouissement de la bruyère.

Ici, nous avons rompu avec la tradition en appliquant soit un fraisage en plein à $10 \mathrm{~cm}$ de profondeur (technique peu couteuse utilisée dans une partie de la plantation de pins de Corse), soit un fraisage par bandes de $50 \mathrm{~cm}$ de largeur après sous-solage à $60 \mathrm{~cm}$ de profondeur suivant l'axe des bandes.

Ces deux méthodes ont, en commun, l'avantage de mélanger les horizons organiques et minéraux, d'accélérer la minéralisation, d'incorporer avec homogénéité les engrais au sol sur une faible profondeur et d'éliminer temporairement la végétation secondaire. Le sous-solage particulier à l'une des deux a pour but de rompre l'horizon ferrique imperméable décrit précédemment et de drainer le sol.

Le labour en plein n'ayant pas été retenu au nombre des traitements étudiés, nous ne sommes pas en mesure de le comparer aux deux méthodes adoptées dans nos essais. Nous nous bornons simplement à confronter ces dernières entre elles et ce, exclusivement dans l'expérience sur pin de Corse.

Il ressort de cette confrontation qu'aucune des deux techniques envisagées, ne prévaut sur l'autre, du moins jusqu'à présent. En effet, les arbres étant très jeunes, leur système radiculaire est encore peu développé de sorte que le travail du sol en profondeur (sous-solage) ne peut avoir d'incidence sur la croissance. Dans cette hypothèse, on ne peut en attendre un effet positif que plus tard: ceci confirmerait les observations d'autres auteurs (Clichroot et Remy, 1955; Galoux, 1954).

Cependant, nous avons pu observer dès maintenant les faits suivants qui pourraient avoir des répercussions sur l'avenir:

1) Après le fraisage en plein, la jeune bruyère colonise le terrain et forme un couvert uniforme qui attire particulièrement les coqs de bruyère. Ceux-ci ébourgeonnent les pins de petites dimensions. A l'appui de cette observation faite sur le terrain, on constate qu'en général le pourcentage de plants qui végètent 
(pins sans pousses terminales) est plus élevé dans les parcelles fraisées en plein que dans celles fraisées par bandes, puis soussolées. Ceci ressort du tableau XIII où l'on a $26,8 \%$ des plants relevant du premier cas, contre $17,9 \%$ des plants appartenant au second.

2) Les champs d'expérience font partie d'un ensemble de 50 ha appartenant à la commune de Mechelen-aan-de-Maas. Ce territoire a été mis à la disposition du Centre de Bokrijk à des fins expérimentales. En vue d'essais futurs, le Centre a effectué sur toute son étendue un sous-solage suivant des axes distants de $1 \mathrm{~m}$ ou de $1,50 \mathrm{~m}$ selon les cas. Depuis lors, ces terrains qui étaient inondés chaque année en hiver, se sont avérés bien drainés. Ils sont en effet devenus accessibles aux véhicules, même en période de pluie prolongée. Il est intéressant de constater que ce n'est pas le cas du demi champ d'expérience sur pin de Corse qui n'a subi qu'un simple fraisage en plein. Et l'on peut imaginer que dans ce cas, la situation eut été plus mauvaise encore si les alentours eux-mêmes n'avaient pas bénéficié d'un assainissement par sous-solage.

\section{3 - Ecartements de plantation.}

L'écartement traditionnel est de $1 \mathrm{~m} \times 1 \mathrm{~m}$. Il est dicté par des raisons d'ordre cultural et technologique: les arbres doivent disposer dans leur première jeunesse d'un espace suffisant pour se fortifier et atteindre l'état de massif vers l'âge de 8 à 10 ans; ces conditions favorisent leur accroissement en hauteur et dans la suite, facilitent l'élagage naturel, donnent lieu à des fûts de forme plus cylindrique et permettent de produire du bois de meilleure qualité (PERRIN, 1952 et 1954; Poskin, 1939).

L'écartement de plantation d'une essence donnée ne dépend donc pas exclusivement de son comportement spécifique, mais aussi du substrat sur lequel on la cultive: en principe, plus celui-ci est riche, meuble et profond, moins la plantation doit être dense. C'est pourquoi nous avons jugé intéressant de prévoir trois écartements plutôt qu'un seul $(1 \mathrm{~m} \times 1 \mathrm{~m}, 1 \mathrm{~m} \times 2 \mathrm{~m}, 2 \mathrm{~m} \times 2 \mathrm{~m})$ dans nos essais où le sol est amélioré par des amendements et un ameublissement rationnels.

Si au terme de nos essais, il s'avérait que l'on ptisse adopter en pratique un écartement plus large que d'habitude, on réduirait sensiblement l'investissement au moment de la plantation. De plus, l'économie ainsi réalisée compenserait largement les frais de fumure et de travail du sol. Enfin, ce procédé permettrait peut-être de réduire ou de modifier les premiers produits d'éclaircie dont la valeur marchande est actuellement dérisoire ou nulle.

Mais comme l'indiquent les tableaux X et XIX, les écartements n'ont, jusqu'à présent, aucun effet sur la croissance des pins, ce qui 
s'explique par le très jeune âge de nos plantations expérimentales. II faudra sans doute attendre au moins que le massif soit formé pour tirer les premières conclusions et dans le cas du pin de Corse, il est à craindre qu'elles ne soient faussées, car les plants n'ont pas été calibrés et sont particulièrement hétérogènes.

\section{BIBLIOGRAPHIE}

Bruxisg (D.). - Ueber die Wirkung von Pflanzennährstoffen anf das Wachstum von Kiefern im Jugendstadium. Allg. Forst- u. Jagdstg., S., pp. 168-177, 1961.

Bodeux (G.). - Vues nouvelles sur la sylviculture en Campine, Bull. Soc. For. Belg., n* 6, pp. 217-251, 1949.

Cochran (W.-G.) et Cox (G.-M.). - Experimental Designs. John Wiley and Sons Inc., New York, 1957.

Colpaekt (M.), - Le pin de Corse en Campine (Pinus laricio Poir. Corsicana Loud.).

Bull. Soc. For. Bely., n 6, pp. 263-373, 1937.

Duchaufour $(\mathrm{Ph}$.$) . - Un cas de carence azotée de l'épicéa décelée par le$ diagnostic foliaire.

Rev. for. franc., pp. 128-136, 1957.

Duchavfovr ( $\mathrm{Ph}$.) - - L'utilisation des engrais en forét. Rev. for. franc., pp. 377-392, 1958.

Duchaufouk $(\mathrm{Ph}$, ) et Guisaudead (J.). - Une expérience de chaulage sur humus brut.

An. E.N.E.F, et stat, de rech, et exp., tome XV, fasc, 2, pp. 335-360. 1957.

Duchaufour (Ph.) et Bonneau (M.). - Note sur la physiologie de la nutrition des résineux.

Rev. for, franç., pp. 250-256, 1960.

Cucheroux (E.) et Rrmy (E.). - Le problème du reboisement en BasseSaxe et quelques questions connexes.

Bull, Soc, For, Belg., n* 4, pp. 177-198, 1955.

Fisher (R.-A.) et Yates (F.). - Statistical Tables for Biological, Agricultural and Medical Research,

Oliver and Boyd, Edinburgh, 1957.

Franz (H.). - Die Walddūngung im Lichte der Bodenbiologie. Allg. Forstsfg., $\mathrm{n}^{\circ}$ 25-26, pp. 321-323, München, 1956.

Franz (H.). - Der derseitige Stand unseres Wissens auf dem Gebiete der Walddüngung.

Allg. Forsts., n* 5-6, pp. 58-63, Wien, 1957.

GaLoux (A.). - Les grands problèmes de sylviculture en Belgique. Bull, Soc, For, Belg., n² 2, pp. 90-113, 1952.

GaLoux (A.). - La fertilisation minérale en sylviculture. Stat. de rech. de Groenendaal, Travaux, Série B, $\mathrm{n}^{\circ} 16,1954$.

Guisaudeau (J.), Illey (G.), Mauge (J.-P.), Dumas (F.). - Essai de fertilisation minérale sur pin maritime à Mimizan (Landes).

Ann. E.N.E.F. et Stat. Rech. et Exp. for., Nancy, Tome XX, fase, 1, pp. $1-71,1963$.

Hurgh (A.). - Travail du sol à l'établissement de bois en Campine. Bull. Soc, For. Belg., n* 3, pp. 137-160, 1957.

Lastekerts (D.). - Bodems uit de Limburgse Kempen. Pédologie, VIII, pp. 224-230, Gent, 1958.

Laмнектs (D.). - Opname en export van minerale voedingselementen door Korsikaanse den (Pinus nigra Arnold).

Pídologie, XII, pp. 101-104, Gent, 1962. 
Leinundgut (H.) et Kreutzer (R.). - Untersuchungen ūber die Wurzelkonkurrenz. 1. Mitteilung über den Vorwald.

Mitt. Schtweis. Anst. forstl. Vers. W., 34, pp. 361-398, Zürich, 1958.

I.xxтos (L.). - The growth and mineral nutrition of Spruce and Pine in Heatland plantation.

Imp. For. Inst. Univ, of Oxford, 1954.

NFF (L.). - Etude d'une population de larves de Retinia buoliana (Schiff.). Z. angew. Ent., $44, \mathrm{n}^{\circ} 2$, Dp. 167-186, 1959.

Perrin (H.). - Sylviculture. Tomes I et II, Ecole Nationale des Eaux et Forèts, Nancy, 1952 et 1954.

Poskin (A.). - Traité de Sylviculture. Duculot, Gembloux, 1939.

Ronde. (G.), - Walbodendungung und Lebensgemeinschaft. Bodenfauna. Z. angete. Ent., 47, $\mathrm{n}^{\circ}$ 1, pp. 52-57, 1960.

SchelliNg (J.). - De hoge bosgronden van Midden-Nederland. Bodemkundige studies, $\mathrm{n}^{\circ} 5$, Wageningen, 1960.

SChWERdTFEger (F.). - Die Waldkrankheiten. Verlag Paul Parey, Hamburg und Berlin, S., pp. 314-318, 1957.

SNEDECOR (G.-W.). - Statistical Methods applied to Experiments in Agriculture and Biology. the Iowa State College Press, Ames, Iowa, 1956.

VAN Goor (C.-P.). - Bewerking en vruchtbaarheid van droge bosgronden. Uitvoerige verslagen von het Bosb. Proefsta. T.N.O., verslag $\mathrm{n}^{\circ} 2$, pp. 51-99, Wageningen, 1952.

VAX Gook (C.-P.). - Kaligebrek als oorzaak van gelepuntziekte van groveden (Pinus sylvestris) en Korsikaanse den (Pinus nigra var. Corsicana). Bosb. Proefsta T.N.O., korte meded., $\mathrm{n}^{\circ}$ 25, pp. 21-31, 1956.

Van Mirgroet (M.) et Janssens (F.). - Aufbau und Wachstum von Bestănden der Waldföhre und der Korsikanischen Schwarzföhre in NordBelgien.

Forstw. Cbl., S., pp. 458-468, 1956.

YATES (F.), - The Design and Analysis of Factorial Experiments. Imperial Bureat of Soil Science, Technical Communication, $n^{\circ} 35$, Harpenden, England, 1937. 


\section{RESUMÉ}

Les auteurs décrivent en détail deux expériences distinctes établies en Campine (Belgique) sur podzol à horizon ferrique situé à faible profondeur.

La première a pour but de contróler l'effet d'une fumure complète et de trois écartements $(1 \times 1 \mathrm{~m}, 1 \times 2 \mathrm{~m}, 2 \times 2 \mathrm{~m})$ sur la croissance du pin sylvestre. Le champ d'essai a subi un travail du sol uniforme: fraisage par bandes de $50 \mathrm{~cm}$ de largeur après soussolage à $60 \mathrm{~cm}$ de profondeur suivant l'axe des bandes.

La seconde contróle chez le pin de Corse l'effet des mêmes facteurs, fumure et écartements, et compare le sous-solage à un fraisage en plein à $10 \mathrm{~cm}$.

Les doses d'engrais employées à l'hectare sont respectivement: 2 tonnes de marne et 1 tonne de scories de déphosphoration, épandues en plein avant le travail du sol, $200 \mathrm{~kg}$ de chlorure de potasse $20 \%$ et $100 \mathrm{~kg}$ de nitrate d'ammoniaque $17 \%$ épandus un an après la plantation.

Les parcelles, réparties selon un dispositif en bloc à 3 répétitions ont des dimensions communes, $25 \times 25 \mathrm{~m}$, pour les deux expériences.

Dans l'expérience sur pin de Corse, le plan factoriel $(3 \times 2) \times 2$ est arrangé en "half-plaid square " pour répondre aux impératifs d'ordre technique que posent deux modes de travaux du sol que l'on ne peut pratiquement exécuter que sur des surfaces assez grandes.

La plantation a été effectuée en avril 1958 suivant la méthode de plantation en fente avec des plants de 2 ans (S1R1) d'origine connue.

L'interprétation des mensurations effectuées en juin-juillet 1962 (hauteur totale 1962, longueur des pousses annuelles 1960 et 1961) ne conduit à des conclusions que dans des limites de temps très étroites.

L'effet favorable de l'engrais sur la croissance des pins se narque à partir de 1960, soit deux ans après la plantation. Le taux de mortalité résultant de la transplantation est cependant à peine lié au traitement. 
L'accroissement relatif de rendement dû à la fumure s'affirme d'année en année chez le pin sylvestre et plus encore chez le pin de Corse.

En ce qui concerne le travail du sol, aucune des deux techniques appliquées ne prévaut jusqu'à présent, ce qui pourrait s'expliquer par le système radiculaire encore insuffisamment développé.

Le fraisage en plein favorise l'extension de la jeune bruyère, ce qui attire particulièrement les petits tétras. Ils ébourgeonnent les pins, d'où le nombre de plants végétants (sans pousses terminales) plus élevé dans les parcelles fraisées en plein (26,8\% contre $17,9 \%)$.

Le sous-solage a eu pour effet de drainer les parcelles traitées. Les écartements n'ont jusqu'à présent aucun effet décelable sur la croissance des pins. 


\section{SUMMARY}

The afforestation of the "Kempen " (The Campine) has been planned extensively and directed to the mining industry since the first artificial plantation of needle-leaved trees. The afforestation mainly consists of monocultures of the Scots Pine (Pinus sylvestris L.), completed with the Corsican Pine (Pinus nigra Arnold var. Corsicana) during the last decades.

In addition to the insect-nuisance and forest-fire which continually infest this traditional afforestation, the low yields of many plantations don't meet the requirements of modern economy. In consequence of the rising cost-price as well of the woodlands as of the plantation and management, the risks for extensive afforestation are too high.

The a Centre de Recherche en Biologie Forestière " at Bokrijk takes all these elements into account and aims at

- a better knowledge of the extensive exploitation methods by rational use of the soil (tillage, fertilizing, selection),

- adapted plantation methods (methods of planting, distance, mixture of species, rhythm and intensivity of thinning).

This is first of all done for traditional species: the Scots Pine (Pinus sylvestris I.) and the Corsican Pine (Pinus nigra Arnold var. Corsicana) for both of them are best adapted to the predominantly dry sandy soil. The results above mentioned are the first applications of this task.

The test-plan and the statistical working-up of the results were done by the "Bureau de Biométrie $n$.

The aim of the test with the Pinus sylvestris L. is to get a better knowledge of the influence of complete fertilizing on the growth of the above mentioned conifer, at interaction of three distances $(1 \times 1 \mathrm{~m}, 1 \times 2 \mathrm{~m}, 2 \times 2 \mathrm{~m})$.

The experimental field was homogeneously furrowed in $50 \mathrm{~cm}$ wide bands and broken up to a depth of $60 \mathrm{~cm}$ following the axes of the bands. Distance between axes is $1 \mathrm{~m}$.

In a second experimental field, planted with Corsican Pine (Pinus nigra Arnold var. Corsicana), we studied the influence of a fertilizing (the same as for the Scots Pine) at three distances. 
We can summarize this experiment:

- fertiliser $(+)$ and $(-)$,

- distances $(1 \times 1 \mathrm{~m}, 1 \times 2 \mathrm{~m}, 2 \times 2 \mathrm{~m})$,

- tillage: furrowing in bands to a depth of $50 \mathrm{~cm}$ and breaking open of the subsoil to a depth of $60 \mathrm{~cm}$; complete furrowing to a depth of $10 \mathrm{~cm}$ without breaking open the subsoil.

The two tests are done on the same heathland of the Meuse-tableland at Mechelen-aan-de-Maas at an altitude of 90 metres.

The soil consists of sandy sedimentary deposits of the Meuse on an undeep, little permeable substratum of clayish sand. The development of the profile is of the type " podzol n with a humusB-horizon. During heavy rainfall there is a temporary water-table on the substratum. The a podzol $n$ built up in these circumstances of temporary water-surplus is marked out by a strongly hardened B-horizon with a clearly developed iron pan on the underside of the humus-B-horizon. That brings about a very unfavourable consumption of water (1).

Moreover the soil is sour, very poor and as well physically as biologically of low quality.

The method of tillage and the choice of fertilizers are adapted to the characteristics of the ground.

The fertilizing happened in two phases: two tons of marl and one ton of scoriae were spread before the tillage, and $200 \mathrm{~kg}$ of potassium $20 \%$ and $100 \mathrm{~kg}$ of ammonia-nitrate per hectare were spread one year later.

The trees were planted in the spring of 1958 with two-yearold plants (S1 R1) of know origin.

The plots are all of the same size, squares $25 \times 25 \mathrm{~m}$, so that the number of trees per plot depends on the distance between the plants.

The test with Pinus sylvestris L., in which the influences of fertilizing and distance are studied, is conceived as a factorial test, $2 \times 3$ blocks being randomised. The six combinations of methods are spread at random in each of the three blocks.

The test with Pinus nigra Arnold var. Corsicana is also a factorial test $(3 \times 2) \times 2$, in which we have two tillages. It is conceived as a " half-plaid square $n$, in which we can't measure separately the specific influences of the breaking open of the ground.

The measurings were done in the autumn of 1962: the total height of the trees in 1961 and the length of the yearly shoots of 1960 and 1961. This enables us to find the total height for 1959 and 1960 .

The separate measurings according to the various kinds of heights are generally spread symmetrically for Pinus sylvestris L., so pro-

(1) In this way, the B-horizons build a secondary \& Staunässe s so that the field, after a heavy rainfall, is submerged after rather a short time. 
bably according to a Gatuss-curve. Notwithstanding differences in number the averages of the test-fields can be compared: there arc enough trees to expect a homogeneous variability between the measurings of the whole test (test-tables IV and VIII).

As there were not any top-shoots in 1960 and 1961 due to the damage caused by black grouse, the spreading of the total heights of Pinus nigra Arnold var. Corsicana was broken and is no longer symmetrical.

The statistical analysis was also applied to comparable averages as for Pinus sylvestris L.

This study enables us to draw the following conclusions.

- The fertilizing has a favourable influence on the growth of the trees from the second year on already. Nortwithstanding this restult the loss caused by the plantation is almost independent of the work, as may be concluded from the following table which gives the percent of dead or dying plants.

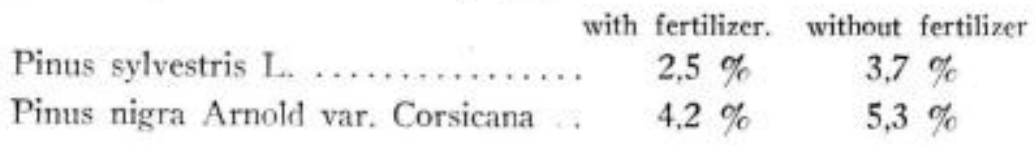

- The relative increase of the yield due to fertilizing becomes gradually clearer and clearer for the Corsican Pine than for the ordinary pine. In 1959, this value was of the same order for the two species $(2.4$ and $2,30 \%)$. In 1961, the growth of the Corsican Pine was more than the double of this of Pinus sylvestris L. (viz, 20,8 and $9,4 \%$ ).

- The two methods of tillage don't seem to be different as to their specific influence on the growth of the trees. The presumahle reason is the up-to-now little extended root-system. which is still superficial.

Moreover, a complete furrowing favours the colonisation by yonng heath, which attracts black grouse. They damage the young plantations and their damage is directly connected with the method of tillage.

- Complete furrowing gives $26.8 \%$ of pines without end-buds, furrowing in bands gives $17,9 \%$ of pines without end-buds.

- Wherever the soil was broken the surface is drained. The plots that were worked in this way, can always be reached by car, even after long periods of rainfall, which can't he done where the ground was only furrowed superficially.

- Up-to-now the various test-distances have no influence on the growth of both species, which is the consequence of the recent establishment of the test. 


\section{ZUSAMMENFASSUNG}

Die Verfasser beschreiben in einzelnen zwei verschiedene Versuche im Kempenland (Belgien) auf Podsol mit untiefem Anreicherungshorizont.

Der erste Versuch hat zum Zweck die Wirkung einer vollständigen Düngung und dreier Entfernungen $(1 \times 1 \mathrm{M}, 1 \times 2 \mathrm{M}, 2 \times 2 \mathrm{M})$ zu kontrollieren auf den Wuchs der Kiefern.

Die Versuchsanlage wurde überall auf dieselbe Weise bearbeitet: mit dem Rotovator in Streifen von $50 \mathrm{Cm}$ breit nach einer Unterschichtbearbeitung von $60 \mathrm{Cm}$ tief der Achse der Streifen folgend.

Der zweite Versuch kontrolliert bei der Corsische Kiefer dic Wirkung derselben Faktoren, Düngung und Entfernung, und vergleicht die Unterschichtbearbeitung mit einer Oberflächebearbeitung auf $10 \mathrm{Cm}$.

Die Düngerdosissen per Hektar sind: 2 Tonnen Mergel und 1 Tonne Thomasschlacken, gestreut vor der Bodenbearbeitung, 200 Kg Kaliumchloride $20 \%$ und $100 \mathrm{Kg}$ Ammoniumnitrat $17 \%$, ein Jahr nach der Bepflanzung gestreut.

Die Blockanlagen mit drei Wiederholungen haben gemeinsame Abmessungen von $25 \times 25 \mathrm{M}$ für beide Versuche,

Bei dem Versuch der Corsische Kiefer wurde der faktorielle Plan $(3 \times 2) \times 2$ angelegt wic " half-plaid square " um allen technischen Anforderungen zu genügen welche beide Bodenbearbeitungen fragen und welche praktisch nur auf ziemlich grosse Anlagen veranstaltet werden können.

Die Bepflanzung fand statt in April 1958 nach der Bepflanzungsmethode mit zweijăhrigen Pflanzen von gekannter Herkunft.

Die Auslegung der in Juni und Juli 1962 gemachten Messungen (Gesamthöhe 1962. Länge der jährlichen Schösslinge 1960 und 1961 ( führt nur in sehr beschränktem Zeitraum zu einem Entschluss.

Die günstige Wirkung der Düngung auf den Wuchs der Kiefern zeigt sich seit 1960, das heisst 2 Jahre nach der Bepflanzung.

Der Ausschuss infolge der Verpflanzung ist jedoch mit der Behandlung kaum verbunden. 
Die relative Errtragserhöhung infolge der Düngung bestātigt sich nach jedem Jahr bei der Kiefer und mehr noch bei der Corsische Kiefer.

Bei der Bodenbearbeitung überwiegt bisher keine der beiden Techniken, was sich erklären lässt aus dem ungenügend entwickelten Wuchs der Wurzehn.

Die Bearbeitung mit dem Rotovator begünstigt die Entwicklıng der jungen Heide, was besonders die kleinen Birkhähne anzieht.

Sie beissen die Knospen ab. Daher findet man in den mit dem Rotavator bearbeiteten Anlagen eine grössere Zahl vegetierende Pflanzen (ohne Endschlösslinge) : $26,8 \%$ gegen $17,9 \%$.

Die Unterschichtbearbeitung hat als Folge die behandelten Anlagen zu drainieren.

Die Entfernungen haben bisher keine sichtbare Folge auf den Wuchs der Kiefern. 